\title{
GROUP FILTERS AND IMAGE PROCESSING
}

\author{
Richard Tolimieri and Myoung An \\ Prometheus Inc. \\ Newport, Rhode Island U.S.A. \\ myoung@prometheus-inc.com
}

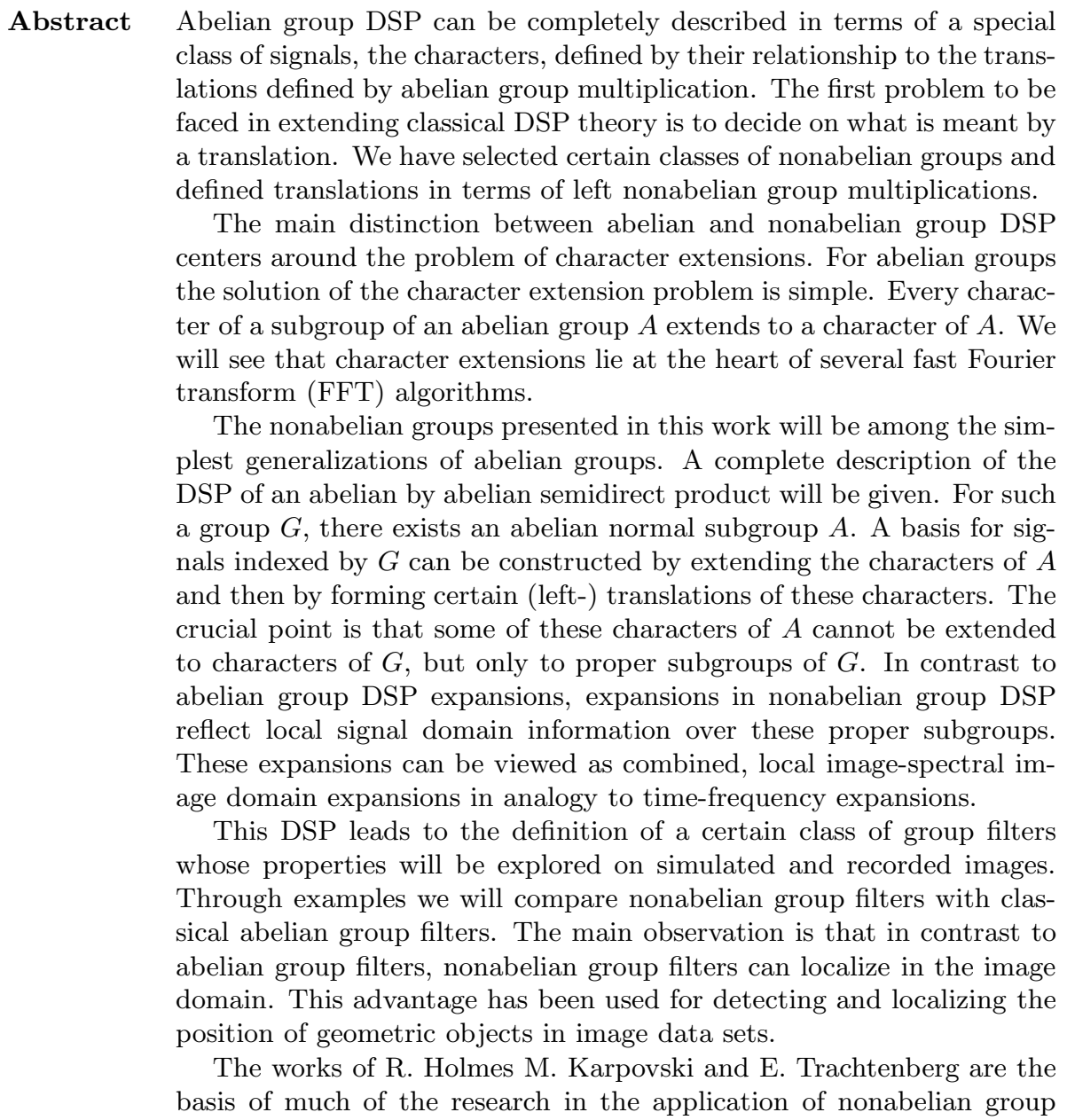


harmonic analysis to DSP, especially in the construction of group transforms and group filters. Similar ideas in coding theory have been introduced by F. J. MacWilliams.

During the last ten years considerable effort has taken place to extend the range of applicability of nonabelian group methods to the design of new filters and spectral analysis methodologies, as an image processing tool. Accompanying these application efforts, there has been extensive development of algorithms for computing Fourier transforms over nonabelian groups generalizing the classical FFT.

Keywords: finite group harmonic analysis, group filters, image domain locality.

\section{Introduction: Classical Digital Signal Processing}

There are two disjoint themes in this work: the fundamental role played by translations of data in all aspects of digital signal processing (DSP) and the role of group structures on data indexing sets in defining these translations.

The set of integers paired with addition modulo $N$ is the simplest finite abelian group. The term abelian refers to the fact that addition modulo $N$ is a commutative binary operation. We denote this group by $\mathbf{Z} / N$ and call it the group of integers modulo $N$.

Denote by $\mathcal{L}(\mathbf{Z} / N)$ the space of all complex valued functions on $\mathbf{Z} / N$. $\mathcal{L}(\mathbf{Z} / N)$ is the space of $N$-point data sets.

For $y \in \mathbf{Z} / N$, the mapping $T(y)$ of $\mathcal{L}(\mathbf{Z} / N)$ defined by

$$
(T(y) f)(x)=f(x-y), \quad f \in \mathcal{L}(\mathbf{Z} / N), x \in \mathbf{Z} / N,
$$

is a linear operator of $\mathcal{L}(\mathbf{Z} / N)$ called translation by $y$.

For $g \in \mathcal{L}(\mathbf{Z} / N)$, the mapping $C(g)$ of $\mathcal{L}(\mathbf{Z} / N)$ defined by

$$
C(g)=\sum_{y \in \mathbf{Z} / N} g(y) T(y)
$$

is a linear operator of $\mathcal{L}(\mathbf{Z} / N)$ called convolution by $g$. If $f \in \mathcal{L}(\mathbf{Z} / N)$, then

$$
(C(g) f)(x)=\sum_{y \in \mathbf{Z} / N} f(x-y) g(y), \quad x \in \mathbf{Z} / N .
$$

\subsection{Fourier Analysis}

Fourier analysis provides the tools for the detailed analysis and construction of translation-invariant subspaces of $\mathcal{L}(\mathbf{Z} / N)$.

For $y \in \mathbf{Z} / N$, define $\chi_{y} \in \mathcal{L}(\mathbf{Z} / N)$ by

$$
\chi_{y}(x)=e^{2 \pi i \frac{y x}{N}}, \quad x \in \mathbf{Z} / N .
$$


The collection

$$
\left\{\chi_{y}: y \in \mathbf{Z} / N\right\}
$$

is a basis of $\mathcal{L}(\mathbf{Z} / N)$ called the exponential basis.

A mapping $\chi: \mathbf{Z} / N \longrightarrow \mathbf{C}^{\times}$is called a character of $\mathbf{Z} / N$ if $\chi$ satisfies

$$
\chi(x+y)=\chi(x) \chi(y), \quad x, y \in \mathbf{Z} / N .
$$

$\mathbf{C}^{\times}$denotes the set of nonzero complex numbers. Direct computation shows that every exponential function is a character of $\mathbf{Z} / N$. Eventually, we will show that the collection of characters and exponential functions of $\mathbf{Z} / N$ coincide.

We want to show that the characters of $\mathbf{Z} / N$ can be defined in terms of the translations of $\mathcal{L}(\mathbf{Z} / N)$.

Suppose $\mathcal{M}$ is a collection of linear operators of a vector space $V$. For $M \in \mathcal{M}$, a nonzero vector $v$ in $V$ is called an $M$-eigen vector if there exists $\alpha \in \mathbf{C}$ such that

$$
M v=\alpha v .
$$

We call $\alpha$ the eigen value of $M$ corresponding to the eigen vector $v$. A nonzero vector $v$ in $V$ is called an $\mathcal{M}$-eigen vector if $v$ is an $M$-eigen vector for all $M \in \mathcal{M}$. In this case, there exists a mapping $\alpha: \mathcal{M} \longrightarrow \mathbf{C}$ such that

$$
M v=\alpha(M) v, \quad M \in \mathcal{M} .
$$

A basis of $V$ is called an $\mathcal{M}$-eigen vector basis if every vector in the basis is an $\mathcal{M}$-eigen vector. Equivalently, a basis for $V$ is an $\mathcal{M}$-eigen vector basis if and only if the matrix of every $M \in \mathcal{M}$ with respect to the basis is a diagonal matrix.

THEOREM 1 If $\chi$ is a character of $\mathbf{Z} / N$, then $\chi$ is a $T(\mathbf{Z} / N)$-eigen vector.

THEOREM 2 If $f$ is a $T(\mathbf{Z} / N)$-eigen vector and

$$
T(y) f=\alpha(y) f, \quad y \in \mathbf{Z} / N,
$$

then $\alpha$ is a character of $\mathbf{Z} / N$ and

$$
f(y)=\alpha(-y) f(0)=\alpha(y)^{*} f(0), \quad y \in \mathbf{Z} / N .
$$

Proof

$$
f(x-y)=\alpha(y) f(x), \quad x, y \in \mathbf{Z} / N .
$$

Setting $y=0$,

$$
f(x)=\alpha(0) f(x), \quad x \in \mathbf{Z} / N .
$$


Since $f \neq 0, \alpha(0)=1, \alpha$ is a character of $\mathbf{Z} / N$. Setting $x=0$ and replacing $-y$ by $y$ completes the proof.

TheOREM 3 If $\chi$ is a $T(\mathbf{Z} / N)$-eigen vector and $\chi(0)=1$, then $\chi$ is a character of $\mathbf{Z} / N$.

By Theorems 1 and 3 the characters of $\mathbf{Z} / N$ are uniquely characterized as the $T(\mathbf{Z} / N)$-eigen vectors taking the value 1 at the origin of $\mathbf{Z} / N$.

We will now complete the picture and show that the characters of $\mathbf{Z} / N$ form a $T(\mathbf{Z} / N)$-eigen vector basis.

Linear algebra theorem If $\mathcal{M}$ is a commuting family of linear operators of a vector space $V$ and each $M \in \mathcal{M}$ has finite order, then there exists an $\mathcal{M}$-eigen vector basis.

THEOREM 4 The set of characters of $\mathbf{Z} / N$ is a $T(\mathbf{Z} / N)$-eigen vector basis.

Proof Choose a $T(\mathbf{Z} / N)$-eigen vector basis

$$
\left\{\chi_{0}, \ldots, \chi_{N-1}\right\}
$$

consisting of characters of $\mathbf{Z} / N$. We will show that if $\chi$ is character of $\mathbf{Z} / N$, then $\chi=\chi_{n}$, for some $n, 0 \leq n \leq N-1$. Write

$$
\chi=\sum_{n=0}^{N-1} \alpha(n) \chi_{n}, \quad \alpha(n) \in \mathbf{C}, 0 \leq n \leq N-1 .
$$

Without loss of generality, assume $\alpha(0) \neq 0$. For $y \in \mathbf{Z} / N$, since

$$
T(y) \chi=\chi(-y) \chi=\sum_{n=0}^{N-1} \alpha(n) \chi_{n}(-y) \chi_{n},
$$

we have

$$
\chi(-y) \alpha(n)=\chi_{n}(-y) \alpha(n), \quad 0 \leq n \leq N-1 .
$$

Setting $n=0$, for $y \in \mathbf{Z} / N$,

$$
\chi(-y)=\chi_{0}(-y)
$$

implying $\chi=\chi_{0}$, completing the proof.

\section{Abelian Group DSP}

Suppose $A$ is an abelian group and $\mathcal{L}(A)$ is the space of all complex valued functions on $A$. 


\subsection{Translations}

For $y \in A$, the mapping $T(y)$ of $\mathcal{L}(A)$ defined by

$$
(T(y) f)(x)=f\left(y^{-1} x\right), \quad x \in A, f \in \mathcal{L}(A),
$$

is a linear operator of $\mathcal{L}(A)$ called translation by $y$.

For $g \in \mathcal{L}(A)$, the mapping $C(g)$ of $\mathcal{L}(A)$ defined by

$$
C(g)=\sum_{y \in A} g(y) T(y)
$$

is a linear operator of $\mathcal{L}(A)$, called convolution by $g$.

By definition

$$
f * g(x)=\sum_{y \in A} f(y) g\left(y^{-1} x\right), \quad x \in A .
$$

TheOREM 5 Convolution is a commutative algebra product on $\mathcal{L}(A)$.

The algebra formed by $\mathcal{L}(A)$ paired with convolution product is called the convolution algebra over $A$.

\section{$2.2 \quad$ Fourier Analysis}

Suppose $\mathbf{C} A$ is the group algebra of $A$.

A mapping $\chi: A \longrightarrow \mathbf{C}^{\times}$is called a character of $A$ if $\chi$ satisfies

$$
\chi(x y)=\chi(x) \chi(y), \quad x, y \in A .
$$

A character of $A$ is simply a group homomorphism from $A$ into $\mathbf{C}^{\times}$.

Denote the set of all characters of $A$ by $A^{*}$. Under the identification between $\mathcal{L}(A)$ and $\mathbf{C} A$, every character $\tau$ of $A$ can be viewed as a formal sum in $\mathbf{C} A$

$$
\tau=\sum_{x \in A} \tau(x) x
$$

Multiplication of characters will always be taken in $\mathbf{C} A$.

ExAmple 6 As elements in $\mathbf{C}\left(C_{2} \times C_{2}\right)$, the characters of $C_{2} \times C_{2}$ are the formal sums

$$
\begin{aligned}
\phi_{0,0} & =1+x_{1}+x_{2}+x_{1} x_{2}, \\
\phi_{1,0} & =1-x_{1}+x_{2}-x_{1} x_{2}, \\
\phi_{0,1} & =1+x_{1}-x_{2}-x_{1} x_{2}, \\
\phi_{1,1} & =1-x_{1}-x_{2}+x_{1} x_{2} .
\end{aligned}
$$


The mapping $\tau_{0}: A \longrightarrow \mathbf{C}^{\times}$defined by $\tau_{0}(x)=1, x \in A$, is a character called the trivial character of $A$. As an element in $\mathbf{C} A$,

$$
\tau_{0}=\sum_{x \in A} x
$$

The importance of characters in DSP is a result of their invariance under left multiplication as stated in the following theorem.

Theorem 7 If $y \in A$ and $\tau$ is a character of $A$, then

$$
y \tau=\tau\left(y^{-1}\right) \tau \text {. }
$$

EXAMPLE 8 In $\mathbf{C} C_{N}$, if

$$
\phi_{n}=\sum_{m=0}^{N-1} v^{n m} x^{m}, \quad v=e^{2 \pi i \frac{1}{N}}
$$

then

$$
x^{r} \phi_{n}=\sum_{m=0}^{N-1} v^{n m} x^{m+r}=\sum_{m=0}^{N-1} v^{n(m-r)} x^{m}=v^{-n r} \phi_{n} .
$$

Corollary 1.1 If $f \in \mathbf{C} A$ and $\tau$ is a character of $A$, then

$$
f \tau=\widehat{f}(\tau) \tau,
$$

where

$$
\widehat{f}(\tau)=\sum_{y \in A} f(y) \tau\left(y^{-1}\right)
$$

Theorem 7 implies every character $\tau$ of $A$ is an $L(A)$-eigen vector,

$$
L(y) \tau=y \tau=\tau\left(y^{-1}\right) \tau, \quad y \in A .
$$

The eigen value of the eigen vector $\tau$ of $L(y)$ is $\tau\left(y^{-1}\right)$.

TheOrem $9 A^{*}$ is the unique $L(A)$-eigen vector basis of $\mathbf{C} A$ satisfying the condition that the value of each basis element is 1 at the identity element 1 of $A$.

The Fourier analysis over $A$ will be developed in terms of the properties of the characters of $A$ in $\mathbf{C} A$. 
Theorem 10 If $\tau$ and $\lambda$ are characters of $A$, then

$$
\tau \lambda= \begin{cases}N \tau, & \tau=\lambda \\ 0, & \tau \neq \lambda\end{cases}
$$

A nonzero element $e$ in $\mathbf{C} A$ is called an idempotent if $e^{2}=e$. Two idempotents $e_{1}$ and $e_{2}$ in $\mathbf{C} A$ are called orthogonal if $e_{1} e_{2}=e_{2} e_{1}=0$. In the language of idempotent theory Theorem 10 says that the set

$$
\left\{\frac{1}{N} \tau: \tau \in A^{*}\right\}
$$

is a set of pairwise orthogonal idempotents.

Since $A^{*}$ is a basis of the space $\mathbf{C} A$, we can write

$$
1=\sum_{\tau \in A^{*}} \alpha(\tau) \tau, \quad \alpha(\tau) \in \mathbf{C} .
$$

For any $\lambda \in A^{*}$,

$$
\lambda=\lambda \cdot 1=\sum_{\tau \in A^{*}} \alpha(\tau) \lambda \tau=N \alpha(\lambda) \lambda
$$

and $\alpha(\lambda)=\frac{1}{N}$, proving the following.

THEOREM 11

$$
1=\frac{1}{N} \sum_{\tau \in A^{*}} \tau
$$

A set of pairwise orthogonal idempotents $\mathcal{I}$ in $\mathbf{C A}$ is called complete if

$$
1=\sum_{e \in \mathcal{I}} e .
$$

In the language of idempotent theory Theorems 10 and 11 say that the set

$$
\left\{\frac{1}{N} \tau: \tau \in A^{*}\right\}
$$

is a complete set of pairwise orthogonal idempotents.

Corollary 1.2

$$
\frac{1}{N} \sum_{\tau \in A^{*}} \tau(x)=\left\{\begin{array}{ll}
1, & x=1, \\
0, & x \neq 1,
\end{array} \quad x \in A .\right.
$$


By Corollary 1.1 and completeness every $f \in \mathbf{C} A$ can be written as

$$
f=\frac{1}{N} \sum_{\tau \in A^{*}} f \tau=\frac{1}{N} \sum_{\tau \in A^{*}} \widehat{f}(\tau) \tau .
$$

We call this expansion of $f$ the Fourier expansion of $f$.

$$
\widehat{f}(\tau)=\sum_{y \in A} f(y) \tau\left(y^{-1}\right), \quad \tau \in A^{*},
$$

is called the Fourier coefficient of $f$ at $\tau$.

\subsection{Character Extensions I}

In this chapter we will show that every character of a direct product $A=B \times C$ of abelian groups $B$ and $C$ can be represented as a product in $\mathbf{C} A$ of a character of $B$ with a character of $C$.

More generally, we will relate the characters of a subgroup of an abelian group to the characters of the group through a construction called character extensions. The generalization of this construction to nonabelian groups will occupy a great deal of the later chapters. $A$ is an abelian group throughout this chapter.

\subsection{Direct Products}

Suppose $B$ is a subgroup of $A$ and $\rho$ is a character of $A$. The mapping $\rho_{B}: B \longrightarrow \mathbf{C}^{\times}$defined by

$$
\rho_{B}(x)=\rho(x), \quad x \in B,
$$

is a character of $B$ called the restriction of $\rho$ to $B$. As an element in $\mathbf{C} A$,

$$
\rho_{B}=\sum_{x \in B} \rho(x) x .
$$

Theorem 12 Suppose $A=B \times C$. If $\rho$ is a character of $A$, then $\rho=$ $\rho_{B} \rho_{C}$, the product in $\mathbf{C} A$ of the characters $\rho_{B}$ and $\rho_{C}$ of $B$ and $C$ defined by the restrictions of $\rho$.

By Theorem 12 the character $\rho$ of $A=B \times C$ is represented as a product in $\mathbf{C} A$ of a character of $B$ with a character of $C$. The representation is unique.

Theorem 12 easily extends to direct product factorizations of $A$ having any number of factors.

ExAmple 13 The character

$$
1+x_{1}-x_{2}-x_{1} x_{2}-x_{3}-x_{1} x_{3}+x_{2} x_{3}+x_{1} x_{2} x_{3}
$$


of $C_{2}\left(x_{1}\right) \times C_{2}\left(x_{2}\right) \times C_{2}\left(x_{3}\right)$ is the product

$$
\left(1+x_{1}\right)\left(1-x_{2}\right)\left(1-x_{3}\right)
$$

of the characters $1+x_{1}, 1-x_{2}, 1-x_{3}$ of $C_{2}\left(x_{1}\right), C_{2}\left(x_{2}\right), C_{2}\left(x_{3}\right)$.

Theorem 14 Suppose $A=B \times C$. If $\tau$ and $\lambda$ are characters of $B$ and $C$, then the product $\rho=\tau \lambda$ in $\mathbf{C} A$ is a character of $A$.

Proof By Theorem 7 if $x=u v, u \in B, v \in C$, then

$$
x \rho=(u \tau)(v \lambda)=\tau\left(u^{-1}\right) \lambda\left(v^{-1}\right) \tau \lambda=\rho\left(x^{-1}\right) \rho,
$$

implying $\rho$ is a character of $A$, completing the proof.

\subsection{Character Extensions}

Suppose $B$ is a subgroup of $A$ and $\tau$ is a character of $B$. A character $\rho$ of $A$ is called an extension of $\tau$ if $\tau$ is the restriction of $\rho$ to $B$.

Consider a complete system of $B$-coset representatives in $A$,

$$
\left\{y_{s}: 1 \leq s \leq S\right\}
$$

Every $x \in A$ can be written uniquely as $x=y_{s} z, 1 \leq s \leq S, z \in B$. Suppose $\rho$ is an extension of $\tau$. Then

$$
\rho=\sum_{x \in A} \rho(x) x=\sum_{s=1}^{S} \sum_{z \in B} \rho\left(y_{s} z\right) y_{s} z .
$$

Since $\rho\left(y_{s} z\right)=\rho\left(y_{s}\right) \rho(z)=\rho\left(y_{s}\right) \tau(z)$,

$$
\rho=\left(\sum_{s=1}^{S} \rho\left(y_{s}\right) y_{s}\right)\left(\sum_{z \in B} \tau(z) z\right)=\left(\sum_{s=1}^{S} \rho\left(y_{s}\right) y_{s}\right) \tau,
$$

proving the following result.

TheORem 15 Suppose $B$ is a subgroup of $A$ and $\tau$ is a character of $B$. If $\rho$ is a character of $A$ extending $\tau$, then

$$
\rho=\left(\sum_{s=1}^{S} \rho\left(y_{s}\right) y_{s}\right) \tau \text {. }
$$

We can use Theorem 15 to characterize the characters extending $\tau$. 
TheOREm 16 Suppose $B$ is a subgroup of $A$ and $\tau$ is a character of $B$. $A$ character $\rho$ of $A$ is an extension of $\tau$ if and only if

$$
\rho=\frac{1}{|B|} \tau \rho
$$

Assume for the rest of this section that $B$ is a subgroup of $A$. For a character $\tau$ of $B$ denote by

$$
\operatorname{ext}_{\tau}(A)
$$

the collection of all characters of $A$ extending the character $\tau$ of $B$. We will show that there always exists a character of $A$ extending $\tau$ and describe $\operatorname{ext}_{\tau}(A)$. If $A=B \times C$, then by Theorems 12 and $14 \operatorname{ext}_{\tau}(A)$ is the set of all products in $\mathbf{C} A$ of the form $\tau \lambda$, where $\lambda$ is a character of $C$.

EXAMPLE 17 The characters of $C_{12}(x)$ extending the character

$$
\tau=\sum_{n=0}^{3} v^{n} x^{3 n}, \quad v=e^{2 \pi i \frac{1}{4}},
$$

of $C_{12}\left(x^{3}\right)$ have the form

$$
\rho_{w}=\sum_{n=0}^{11} w^{n} x^{n}, \quad w^{3}=v .
$$

This will be the case if and only if

$$
w=e^{2 \pi i \frac{1}{12}}, e^{2 \pi i \frac{1}{12}} e^{2 \pi i \frac{1}{3}}, e^{2 \pi i \frac{1}{12}} e^{2 \pi i \frac{2}{3}} .
$$

For each such $w$ we can write the corresponding extension $\rho_{w}$ as

$$
\rho_{w}=\tau\left(1+w x+w^{2} x^{2}\right)
$$

which is the factorization of $\rho_{w}$ given in Theorem 15. In this case we also have the direct product factorization,

$$
C_{12}(x)=C_{12}\left(x^{3}\right) \times C_{12}\left(x^{4}\right)
$$

and can write

$$
\rho_{w}=\tau\left(1+u x^{4}+u^{2} x^{8}\right)
$$

where

$$
1+u x^{4}+u^{2} x^{8}
$$

is the character of $C_{12}\left(x^{4}\right)$ defined by $u=w^{4}$. 


\section{Nonabelian Groups}

EXAMPLE 18 The $3 \times 3$ cyclic shift matrix

$$
S_{3}=\left[\begin{array}{lll}
0 & 1 & 0 \\
0 & 0 & 1 \\
1 & 0 & 0
\end{array}\right],
$$

and the $3 \times 3$ time-reversal matrix

$$
R_{3}=\left[\begin{array}{lll}
1 & 0 & 0 \\
0 & 0 & 1 \\
0 & 1 & 0
\end{array}\right]
$$

satisfy the relations

$$
S_{3}^{2}=S_{3}^{-1} \neq I_{3}, \quad, S_{3}^{3}=I_{3}=R_{3}^{2}, \quad R_{3} S_{3} R_{3}^{-1}=S_{3}^{-1} .
$$

Denote by $\mathcal{D}_{6}\left(S_{3}, R_{3}\right)$ the collection of all matrices

$$
\left\{I_{3}, S_{3}, S_{3}^{2} ; R_{3}, S_{3} R_{3}, S_{3}^{2} R_{3}\right\}
$$

By the above relations $\mathcal{D}_{6}\left(S_{3}, R_{3}\right)$ is closed under matrix multiplication. For example

$$
R_{3} S_{3}=S_{3}^{-1} R_{3}=S_{3}^{2} R_{3} .
$$

Since $R_{3} S_{3} \neq S_{3} R_{3}, \mathcal{D}_{6}\left(S_{3}, R_{3}\right)$ is a nonabelian group of order 6 .

In general we have the following result. Assume $N \geq 3$.

TheOREM 19 The $N \times N$ cyclic shift matrix $S_{N}$ and the $N \times N$ timereversal matrix $R_{N}$ satisfy the relations $S_{N}^{n} \neq I_{N}, 0<n<N$, and

$$
S_{N}^{N}=I_{N}=R_{N}^{2}, \quad R_{N} S_{N} R_{N}=S_{N}^{-1} .
$$

The set of all products $\mathcal{D}_{2 N}\left(S_{N}, R_{N}\right)$ of the form

$$
\left\{S_{N}^{n} R_{N}^{j}: 0 \leq n<N, 0 \leq j<2\right\}
$$

is a nonabelian group of order $2 N$.

The group $\mathcal{D}_{2 N}\left(S_{N}, R_{N}\right)$ is a matrix representation of the dihedral group of order $2 N$. 


\subsection{Normal Subgroups}

Abelian group constructions do not automatically extend to nonabelian groups. The identification of a distinguished class of subgroups, the normal subgroups, is one of the most significant insights in the development of nonabelian group theory.

The concept of a normal subgroup of a nonabelian group $G$ is closely related to a certain class of automorphisms, the inner automorphisms of $G$. We will discuss these concepts in this section.

if

Suppose $H$ and $K$ are subgroups of $G$. We say that $K$ normalizes $H$

$$
y H y^{-1}=H, \quad y \in K .
$$

$H$ is a normal subgroup of $G$ if $G$ normalizes $H$.

\subsection{Quotient Groups}

Suppose, to begin with, that $H$ is any subgroup of $G$.

For $y \in G$, the set

$$
y H=\{y x: x \in H\}
$$

is called the left $H$-coset in $G$ at $y$. Right $H$-cosets can be defined in an analogous manner.

TheOREm 20 Two left $H$-cosets are either equal or disjoint.

The collection of left $H$-cosets in $G$ forms a partition of $G$. Denote this collection by $G / H$ and call $G / H$ the quotient space of left $H$-cosets in $G$.

Theorem 21 If $y, z \in G$, then $y H=z H$ if and only if $z^{-1} y \in H$.

A set

$$
\left\{y_{1}, \ldots, y_{R}\right\}
$$

formed by choosing exactly one element in each left $H$-coset is called a complete system of left $H$-coset representatives in $G$.

TheOREm 22 If $H$ is a normal subgroup of $G$, then the product

$$
(y H)(z H)=y z H, \quad y, z \in G,
$$

is a group product on $G / H$ with $H$ as identity element and

$$
(y H)^{-1}=y^{-1} H, \quad y \in G .
$$

The group $G / H$ is called the quotient group of left $H$-cosets in $G$. 


\subsection{Semidirect Product}

Suppose $H$ is a normal subgroup of $G$. We say that $G$ splits over $H$ with complement $K$ if $G=H K$ and $H \cap K=\{1\}$. If $G$ splits over $H$ with complement $K$, then we say that $G$ is the internal semidirect product $H \Varangle K$. The usual argument shows that $G=H \Varangle K$ if and only if every $x \in G$ has a unique representation of the form $x=y z, y \in H$, $z \in K$. In general, the complement $K$ is not uniquely determined.

If $G=H \Varangle K$ and $K$ is a normal subgroup of $G$ then $[H, K]=\{1\}$ and $G$ is the direct product $H \times K$. What is new in the internal semidirect product is the possibility that $K$ acts nontrivially on $H$.

Suppose $H$ and $K$ are groups and

$$
\Psi: K \longrightarrow \operatorname{Aut}(H)
$$

is a group homomorphism. The semidirect product $H \Varangle_{\Psi} K$ is the pair consisting of the cartesian product $H \times K$ and the binary operation

$$
\left(y_{1}, z_{1}\right)\left(y_{2}, z_{2}\right)=\left(y_{1} \Psi_{z_{1}}\left(y_{2}\right), z_{1} z_{2}\right), \quad y_{1}, y_{2} \in H, z_{1}, z_{2} \in K .
$$

The semidirect product $H \Varangle_{\Psi} K$ is a group having identity $(1,1)$ and inverse

$$
x^{-1}=\left(\Psi_{z^{-1}}\left(y^{-1}\right), z^{-1}\right), \quad x=(y, z) \in H \Varangle{ }_{\Psi} K .
$$

\subsection{Examples: Semidirect product constructions}

DSP algorithms will be designed for nonabelian group indexing sets formed mainly by semidirect product constructions. The groups will have the form $A \Varangle H$, where $A$ is an abelian group.

Consider the ring $\mathbf{Z} / N$ of integers modulo $N$. An $m \in \mathbf{Z} / N$ is called a unit if there exists an $n \in \mathbf{Z} / N$ such that $m n=1$ in $\mathbf{Z} / N$. A unit in $\mathbf{Z} / N$ is an invertible element relative to multiplication in $\mathbf{Z} / N$. The unit group $U(N)$ can be characterized as the set of all integers $0<m<N$ such that $m$ and $N$ are relatively prime.

TheOREM 23 The mapping

$$
u \longrightarrow \Psi_{u}, \quad u \in U(N),
$$

is a group isomorphism from $U(N)$ onto Aut $\left(C_{N}(x)\right)$.

The group isomorphism in Theorem 23 establishes a one-to-one correspondence between the subgroups of $U(N)$ and those of $\operatorname{Aut}\left(C_{N}(x)\right)$. Under this identification, we can form $C_{N}(x) \Varangle K$ for any subgroup $K$ of $U(N)$ by the group isomorphism throughout this work. A typical 
point in $C_{N}(x) \Varangle K$ is denoted by $\left(x^{m}, u\right), 0 \leq m<N, u \in K$ with multiplication given by

$$
\left(x^{m}, u\right)\left(x^{n}, v\right)=\left(x^{m+u n}, u v\right), \quad 0 \leq m, n<N, u, v \in K,
$$

where $m+u n$ is taken modulo $N$. Identifying $C_{N}(x)$ with the normal subgroup $C_{N}(x) \times\{1\}$ of $C_{N}(x) \Varangle K$ and $K$ with the subgroup $\{1\} \times K$ of $C_{N}(x) \Varangle K$, we can write

$$
x^{m} u=\left(x^{m}, u\right), \quad 0 \leq m<N, u \in K .
$$

Groups of the form $C_{N}(x) \Varangle K$ will be used in many examples as several DSP concepts are introduced in the following chapters. Although the identification in (1) is unambiguous as a description of an abstract group, we will use the modification,

$$
x^{m} k_{u}=\left(x^{m}, u\right), \quad 0 \leq m<N, u \in U(N),
$$

with $x^{m} k_{u}^{j}=\left(x^{m}, u^{j}\right)$.

The reason for this modification is to avoid any confusion or awkward notation in viewing $C_{N}(x) \Varangle K$ as a subset of the group algebra

$$
\mathbf{C}\left(C_{N}(x) \Varangle K\right) .
$$

For a prime $p$ the unit group $U(p)$ of $\mathbf{Z} / p$ is a cyclic group of order $p-1$ under multiplication modulo $p$. Choosing a generator $y$ for $U(p)$, we can write the elements of $U(p)$ as successive powers of $y$,

$$
1, y, \ldots, y^{p-2}
$$

with $y^{p-1}=1$.

A typical point in $G=C_{p}(x) \Varangle U(p)$ can be written as

$$
x^{m} k_{y}^{j}, \quad 0 \leq m<p, 0 \leq j<p-1,
$$

with $y$ a generator of $U(p)$. Multiplication in $G$ is subject to the relations

$$
x^{p}=1=k_{y}^{p-1}, \quad k_{y} x=x^{y} k_{y} .
$$

Other semidirect products can be constructed replacing $U(p)$ by any subgroup of $U(p)$.

Suppose $N=p_{1} p_{2}$, with $p_{1}$ and $p_{2}$ distinct primes. A number theoretic result asserts that $U(N)=U\left(p_{1}\right) \times U\left(p_{2}\right)$. There exist $y_{1}, y_{2} \in$ $U(N)$ such that every $y \in U(N)$ can be written uniquely as

$$
y=y_{1}^{j_{1}} y_{2}^{j_{2}}, \quad 0 \leq j_{1}<p_{1}-1,0 \leq j_{2}<p_{2}-1,
$$


with $y_{1}^{p_{1}-1}=1=y_{2}^{p_{2}-1}$ and $y_{1} y_{2}=y_{2} y_{1}$.

EXAMPLE 24 An arbitrary element in the group $C_{N}(x) \Varangle U(N), N=$ $p_{1} p_{2}$, can be written uniquely as

$$
x^{m} k_{y_{1}}^{j_{1}} k_{y_{2}}^{j_{2}}, \quad 0 \leq m<N, 0 \leq j_{1}<p_{1}-1,0 \leq j_{2}<p_{2}-1,
$$

with group multiplication subject to the relations

$$
\begin{gathered}
x^{N}=1=k_{y_{1}}^{p_{1}-1}=k_{y_{2}}^{p_{2}-1}, \\
k_{y_{1}} x=x^{y_{1}} k_{y_{1}}, \quad k_{y_{2}} x=x^{y_{2}} k_{y_{2}}, \quad k_{y_{1}} k_{y_{2}}=k_{y_{2}} k_{y_{1}} .
\end{gathered}
$$

Consider the abelian group $C_{N}(x) \times C_{N}(y)$. Under the usual identifications, we can write a typical point as $x^{m} y^{n}, 0 \leq m, n<N$, subject to the relations $x^{N}=1=y^{N}$ and $x y=y x$. We begin our discussion of $\operatorname{Aut}\left(C_{N}(x) \times C_{N}(y)\right)$ by extending the concept of a unit group to matrices.

The set $M(2, \mathbf{Z} / N)$ of all $2 \times 2$ matrices over $\mathbf{Z} / N$ is a ring with respect to matrix addition and matrix multiplication. The arithmetic required for the matrix addition and multiplication is taken in $\mathbf{Z} / N . I_{2}$ denotes the identity matrix in $M(2, \mathbf{Z} / N)$. A matrix $L \in M(2, \mathbf{Z} / N)$ is called a unit if there exists $L^{\prime} \in M(2, \mathbf{Z} / N)$ such that $L L^{\prime}=I_{2}=L^{\prime} L . L^{\prime}$ is called the inverse of $L$ in $M(2, \mathbf{Z} / N)$ and is uniquely determined when it exists. If $L$ is a unit in $M(2, \mathbf{Z} / N)$, we denote by $L^{-1}$ the inverse of $L$. If $L_{1}$ and $L_{2}$ are units, then

$$
\left(L_{1} L_{2}\right)\left(L_{2}^{-1} L_{1}^{-1}\right)=I_{2}=\left(L_{2}^{-1} L_{1}^{-1}\right) L_{1} L_{2}
$$

and $L_{1} L_{2}$ is a unit with inverse $L_{2}^{-1} L_{1}^{-1}$. The set $G L(2, \mathbf{Z} / N)$ of all units in $M(2, \mathbf{Z} / N)$ is a group under matrix multiplication in $M(2, \mathbf{Z} / N)$. We call $G L(2, \mathbf{Z} / N)$ the unit group in $M(2, \mathbf{Z} / N) . G L(2, \mathbf{Z} / N)$ can be characterized as the set of all $L \in M(2, \mathbf{Z} / N)$ such that $\operatorname{det}(L) \in U(N)$. $\operatorname{det}(L)$ is defined by the same formula for complex matrices but with arithmetic operations taken in $\mathbf{Z} / N$.

For

$$
L=\left[\begin{array}{ll}
L_{1} & L_{2} \\
L_{3} & L_{4}
\end{array}\right] \in M(2, \mathbf{Z} / N)
$$

define $\Psi_{L}: C_{N}(x) \times C_{N}(y) \longrightarrow C_{N}(x) \times C_{N}(y)$ by

$$
\Psi_{L}\left(x^{m} y^{n}\right)=x^{L_{1} m+L_{2} n} y^{L_{3} m+L_{4} n}, \quad 0 \leq m, n<N .
$$

$\Psi_{L}$ is uniquely determined by its values on the generators $x$ and $y$,

$$
\Psi_{L}(x)=x^{L_{1}} y^{L_{3}}, \quad \Psi_{L}(y)=x^{L_{2}} y^{L_{4}} .
$$


TheOREM 25 The mapping

$$
L \longrightarrow \Psi_{L}, \quad L \in G L(2, \mathbf{Z} / N)
$$

is a group isomorphism from $G L(2, \mathbf{Z} / N)$ onto $\operatorname{Aut}\left(C_{N}(x) \times C_{N}(y)\right)$.

Under the group isomorphism in Theorem 25, there is a one-to-one correspondence between the subgroups of $G L(2, \mathbf{Z} / N)$ and the subgroups of $\operatorname{Aut}\left(C_{N}(x) \times C_{N}(y)\right)$ and we can form

$$
\left(C_{N}(x) \times C_{N}(y)\right) \Varangle K,
$$

for any subgroup $K$ of $G L(2, \mathbf{Z} / N)$. A typical point is denoted by $\left(x^{m} y^{n}, L\right), 0 \leq m, n<N, L \in K$ with multiplication given by

$$
\left(x^{m} y^{n}, L\right)\left(x^{m^{\prime}} y^{n^{\prime}}, L^{\prime}\right)=\left(x^{m^{\prime \prime}} y^{n^{\prime \prime}}, L^{\prime \prime}\right)
$$

where

$$
m^{\prime \prime}=m+L_{1} m^{\prime}+L_{2} n^{\prime}, \quad n^{\prime \prime}=n+L_{3} m^{\prime}+L_{4} n^{\prime}, L=\left[\begin{array}{cc}
L_{1} & L_{2} \\
L_{3} & L_{4}
\end{array}\right],
$$

and $L^{\prime \prime}=L L^{\prime}$. Under the usual identifications, we can write

$$
x^{m} y^{n} L=\left(x^{m} y^{n}, L\right), \quad 0 \leq m, n<N, L \in K .
$$

As before, to avoid awkward notation in the group algebra setting, we will use the modified identification,

$$
x^{m} y^{n} k_{L}=\left(x^{m}, y^{n}, L\right), \quad 0 \leq m, n<N, L \in G L(2, \mathbf{Z} / N) .
$$

The discussion extends to any number of cyclic group factors as long as each factor has the same order.

\subsection{Nonabelian Group DSP}

The new problem raised by noncommutativity is that the characters no longer form a basis of nonabelian group algebras. As a result, there exist multidimensional irreducible ideals. Throughout $G$ is an arbitrary nonabelian group of order $N$ and $\mathbf{C} G$ is its group algebra.

EXAMPLE 26 The dihedral group $\mathcal{D}_{2 N}=\mathcal{D}_{2 N}\left(x, k_{N-1}\right)$ is the set of products $x^{m} k_{N-1}^{j}, 0 \leq m<N, 0 \leq j<2$, with multiplication subject to the relations

$$
x^{N}=1=k_{N-1}^{2}, \quad k_{N-1} x=x^{-1} k_{N-1} .
$$


The group algebra $\mathbf{C} \mathcal{D}_{2 N}$ is the set of polynomials of the form

$$
\sum_{m=0}^{N-1} \sum_{j=0}^{1} a_{m, j} x^{m} k_{N-1}^{j}, \quad a_{m, j} \in \mathbf{C},
$$

with polynomial multiplication subject to the relations given for the group $\mathcal{D}_{2 N}$.

Example 27 Set $A=C_{N}(x) \times C_{N}(y), G=A \Varangle C_{2}\left(k_{c}\right)$, where $c=$ $\left[\begin{array}{rr}1 & 0 \\ 1 & -1\end{array}\right]$

In Figure 1 we display lines indexed by $G$ and their translations under multiplications by elements from $G$.

Figure 1. An image and its translates in $\mathbf{C} G_{4}, N=64$
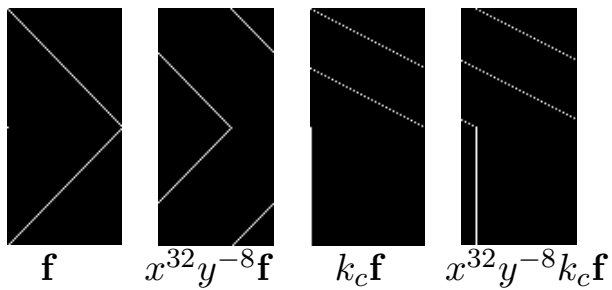

In the following figures, we show the effects of replacing $k_{c}$ by other elements of $G L(2, \mathbf{Z} / N)$ of order 2 .

Set

$$
b=\left[\begin{array}{ll}
0 & 1 \\
1 & 0
\end{array}\right] .
$$

The first image in Figure 2 is the same as in Figure 1. The remaining images are its translates by left multiplications of elements of $A \Varangle C_{2}\left(k_{b}\right)$.

Figure 2. An image and its translates in $\mathbf{C}\left(A \Varangle C_{2}\left(k_{b}\right)\right), N=64$
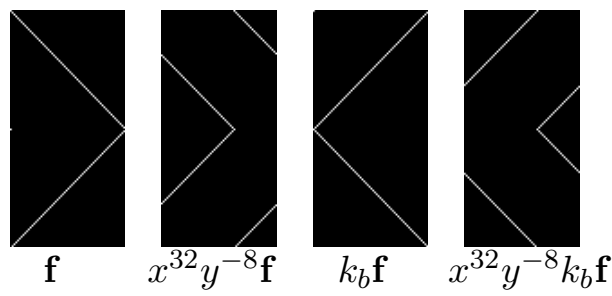
$G_{5}=A \Varangle C_{6}\left(k_{d}\right)$, where $A=C_{N}(x) \times C_{N}(y), N=3 \cdot 2^{K}$ for an integer $K \geq 2$. Set $M=2^{K}$.

$$
d=\left[\begin{array}{cc}
-1 & M+1 \\
M-1 & M
\end{array}\right]
$$

Order $G_{5}$ by

$$
\left[\begin{array}{ccc}
A & A k_{d}^{2} & A k_{d}^{4} \\
A k_{d}^{3} & A k_{d}^{5} & A k_{d}
\end{array}\right]
$$

where $A$ is ordered as before. Figure 3 is an example of translations of data indexed by $G_{5}, M=16$. Starting with the top left image as an element in $\mathbf{C} G_{5}$, the remaining 5 images are obtained by left multiplication by successive powers of $k_{d}$.

\section{Figure 3. Translations by $C_{6}\left(k_{d}\right)$}

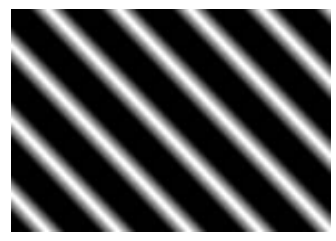

original image

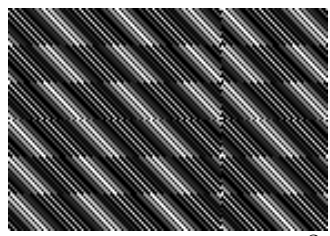

translation by $k_{d}^{3}$

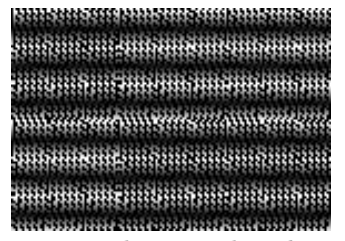

translation by $k_{d}$

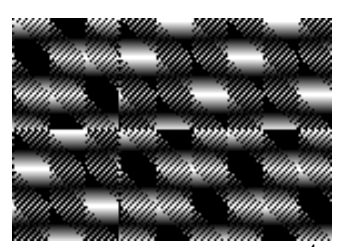

translation by $k_{d}^{4}$

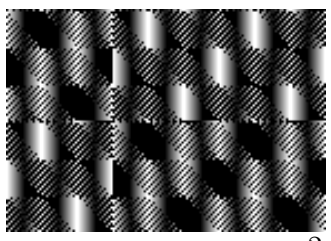

translation by $k_{d}^{2}$

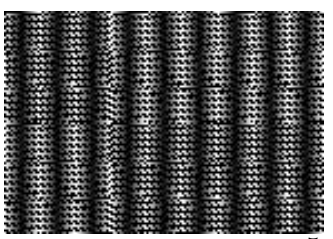

translation by $k_{d}^{5}$

EXAMPLE 28 Varying group structures are placed on an indexing set of data, and products are computed corresponding to the group algebra. $G_{1}$ is the abelian group $C_{2 N}(x)$, where $G_{1}$ is indexed by the successive powers of $x . G_{2}$ is the dihedral group $\mathcal{D}_{2 N}\left(x, k_{N-1}\right) . G_{3}$ is constructed as follows: For $N=2^{M}, M \in \mathbf{Z}, M \geq 2,\left(\frac{N}{2}+1\right)^{2} \equiv 1 \bmod N ; \frac{N}{2}+1$ generates a subgroup of $U(N)$ of order 2 . Set $G_{3}=C_{N}(x) \Varangle\left\{1, k_{\frac{N}{2}+1}\right\}$, $\frac{N}{2}+1 \in U(N)$. The product in $\mathbf{C} G_{3}$ is governed by the relations

$$
x^{N}=k_{\frac{N}{2}+1}=1, \quad k_{\frac{N}{2}+1} x=x^{\frac{N}{2}+1} k_{\frac{N}{2}+1} .
$$


Figure 4. Group algebra products

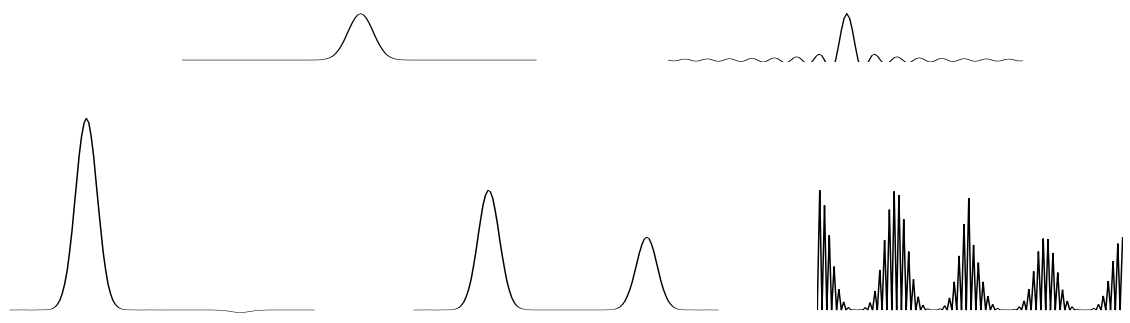

$G_{3}$ is indexed by

$$
1, x, \ldots, x^{N-1} ; k_{\frac{N}{2}+1}, x k_{\frac{N}{2}+1}, \ldots, x^{N-1} k_{\frac{N}{2}+1} .
$$

Note that $G_{2}$ and $G_{3}$ are isomorphic groups.

For $N=64$, the first two plots in Figure 4 are those of 128 points of data. The product in $G_{1}$ is the usual cyclic convolution of two sets of data and displayed in the third plot. The 4th and 5th plots are those of group algebra products in $\mathbf{C} G_{2}$ and $\mathbf{C} G_{3}$.

For $N=128$, each of the groups determines an indexing of data of size 256. The first plots in 5 are those of 256 points of data. The product in $G_{1}$ is the usual cyclic convolution of two sets of data and displayed in the third plot. The remaining plots are those of group algebra products in $\mathbf{C} G_{2}$ and $\mathbf{C} G_{3}$.

EXAMPLE 29 Varying group structures are placed on the indexing set of two-dimensional data, and products are computed corresponding to the group algebra. For $0 \leq m, n<N$, place the lexicographic ordering on the pair $(m, n) . G_{1}$ is the abelian group $C_{N}(x) \times C_{2 N}(y)$ with twodimensional ordering given by

$$
\left(x^{m}, y^{n}\right) ;\left(x^{m}, y^{n+N}\right) \text {. }
$$

$G_{2}$ is the abelian group $\left(C_{N}(x) \times C_{N}(y)\right) \times C_{2}(z)$ with two-dimensional ordering given by

$$
\left(x^{m}, y^{n}\right) ;\left(x^{m}, y^{n} z\right) .
$$

$G_{3}=\left(C_{N}(x) \times C_{N}(y)\right) \Varangle\left\{I_{2}, k_{z}\right\}, z=\left[\begin{array}{cc}-1 & 0 \\ 0 & -1\end{array}\right]$. The ordering of $G_{3}$ is the two-dimensional ordering given by

$$
\left(x^{m}, y^{n}\right) ;\left(x^{m}, y^{n} k_{z}\right) \text {. }
$$


Figure 5. Group algebra products

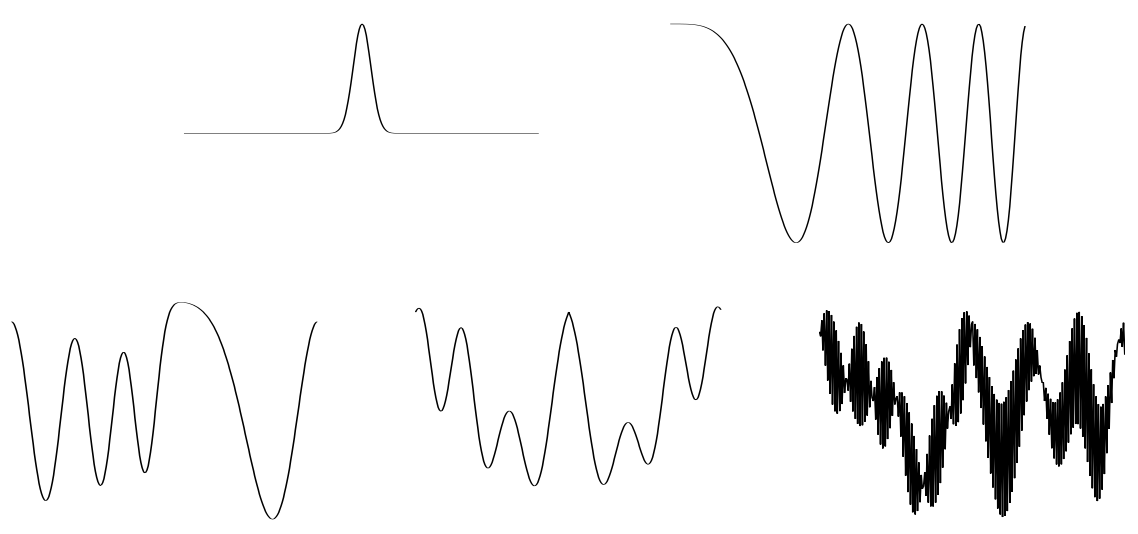

$G_{4}$ is the nonabelian group $\left(C_{N}(x) \times C_{N}(y)\right) \Varangle\left\{I_{2}, k_{u}\right\}, u=\left[\begin{array}{ll}0 & 1 \\ 1 & 0\end{array}\right] \in$ $G L(2, \mathbf{Z} / N)$. The ordering of $G_{4}$ is the two-dimensional ordering given by

$$
\left(x^{m}, y^{n}\right) ;\left(x^{m}, y^{n} k_{u}\right)
$$

For $N=32$, each of the groups determines indexing of data of size $32 \times 64$. Images in Figure 6 are log scaled intensity plots of data of size $32 \times 64$. Images in Figure 7 are convolutions of images in Figure 6 in $\mathbf{C} G_{1}, \mathbf{C} G_{2}, \mathbf{C} G_{3}$ and $\mathbf{C} G_{4}$.

\subsection{Fourier Analysis}

A mapping $\rho: G \longrightarrow \mathbf{C}^{\times}$is called a character of $G$ if it satisfies

$$
\rho(x y)=\rho(x) \rho(y), \quad x, y \in G .
$$

A character of $G$ is simply a group homomorphism of $G$ into $\mathbf{C}^{\times}$. In group representation theory the more general concept of a character of a group representation of $G$ is defined and characters as defined in (2) are called one-dimensional characters. We will not use this general concept. 
Figure 6. $\quad$ Images of size $32 \times 64$
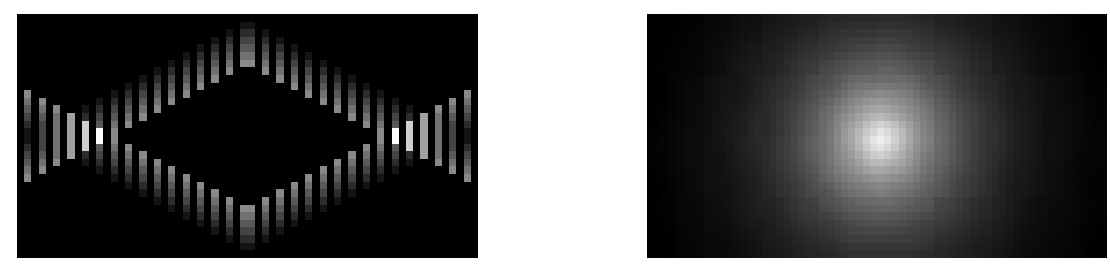

Figure \%. Group algebra products
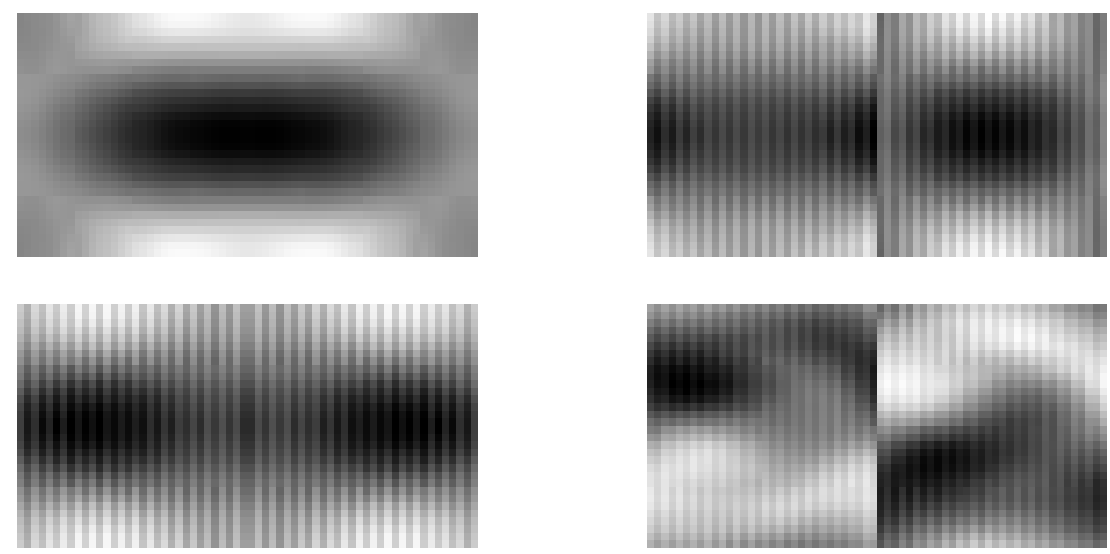

Characters of nonabelian groups are more difficult to describe than in the abelian group case. There always exists a character, the trivial character of $G$, which takes on the value 1 at all points of $G$.

Denote the collection of all characters of $G$ by $G^{*}$. We can view a character $\rho$ of $G$ as a formal sum in $\mathbf{C} G$,

$$
\rho=\sum_{x \in G} \rho(x) x,
$$

and $G^{*}$ as a subset of $\mathbf{C} G$.

ThEOREM 30 Suppose $\rho$ is a character of $G$. If $t \in G$,

$$
t \rho=\rho t=\rho\left(t^{-1}\right) \rho .
$$

Corollary 1.3 Suppose $\rho$ is a character of $G$. If $f \in \mathbf{C} G$, then

$$
f \rho=\rho f=\widehat{f}(\rho) \rho,
$$


where $\widehat{f}(\rho)=\sum_{t \in G} f(t) \rho\left(t^{-1}\right)$.

Theorem 30 leads to an important formula for the product of two characters in $\mathbf{C} G$.

Theorem 31 For characters $\rho$ and $\lambda$ of $G$,

$$
\rho \lambda= \begin{cases}N \rho, & \rho=\lambda, \\ 0 & \rho \neq \lambda .\end{cases}
$$

\subsection{Character Extensions II}

Methods will be developed for constructing characters of nonabelian groups having semidirect product representations. For a group $G=$

$H \Varangle K$, the strategy is to begin with a character $\tau$ of $H$, determine if $\tau$ has an extension to a character of $G$ and if it does, construct the collection of all characters of $G$ extending $\tau$.

An arbitrary character $\tau$ of $H$ does not necessarily extend to a character of $G=H \Varangle K$.

We assume that $H$ is a normal subgroup of $G$ throughout this paper. In Section 3.9, when required, we will explicitly assume that $H$ splits in $G$ and $G=H \Varangle K$.

\subsection{Group Action}

For $y \in G$ and $f \in \mathbf{C} G$, define $f^{y} \in \mathbf{C} G$ by

$$
f^{y}=y f y^{-1}=\sum_{x \in G} f(x) y x y^{-1} .
$$

Two characters $\tau_{1}$ and $\tau_{2}$ of $H$ are said to be conjugate in $G$ if there exists $y \in G$ such that $\tau_{2}=\tau_{1}^{y}$. Conjugacy is an equivalence relation on the set $H^{*}$ of all characters of $H$.

Suppose $\tau$ is a character of $H$ and $G(\tau)$ its centralizer in $G$. Choose a complete system of left $G(\tau)$-coset representatives in $G$,

$$
\left\{y_{s}: 1 \leq s \leq S\right\} \text {. }
$$

Every $y \in G$ can be uniquely written as

$$
y=y_{s} z, \quad 1 \leq s \leq S, z \in G(\tau) .
$$

Define the conjugates $\tau_{s}, 1 \leq s \leq S$, of $\tau$ by

$$
\tau_{s}=y_{s} \tau y_{s}^{-1}, \quad 1 \leq s \leq S .
$$


THEOREM 32 If $\tau$ is a character of $H$, the conjugates $\tau_{s}, 1 \leq s \leq S$, of $\tau$ defined by (3) are distinct characters of $H$ and the set

$$
\left\{\tau_{s}: 1 \leq s \leq S\right\}
$$

is the collection of all conjugates of $\tau$ in $G$.

Proof Suppose $y \in G$ and write $y=y_{s} z, 1 \leq s \leq S, z \in G(\tau)$. Since $G(\tau)$ centralizes $\tau$,

$$
y \tau y^{-1}=y_{s} z \tau z^{-1} y_{s}^{-1}=y_{s} \tau y_{s}^{-1}=\tau_{s}
$$

and every conjugate of $\tau$ in $G$ is of the form $\tau_{s}, 1 \leq s \leq S$. If $\tau_{s}=\tau_{t}$, $1 \leq s, t \leq S$, then

$$
\tau=y_{s}^{-1} y_{t} \tau y_{t}^{-1} y_{s}
$$

This implies $y_{s}^{-1} y_{t} \in G(\tau)$ and $y_{s}=y_{t}$, completing the proof.

By Theorem 31 we have the following corollary.

Corollary 1.4 If $1 \leq s, t \leq S$ and $s \neq t$, then $\tau_{s} \tau_{t}=\tau_{t} \tau_{s}=0$.

The characters $\phi_{n}, 0 \leq n<M$, of $C_{M}(x)$ are defined by

$$
\phi_{n}\left(x^{m}\right)=v^{n m}, \quad 0 \leq m<M, v=e^{2 \pi i \frac{1}{M}} .
$$

If $0 \leq n<N$ and $m \in U(M)$, then since

$$
\phi_{n}^{k_{m}}(x)=\phi_{n}\left(x^{m}\right)=\phi_{n m}(x),
$$

the set

$$
\left\{\phi_{n m}: m \in U(M)\right\}
$$

is the set of conjugates of $\phi_{n}$ in $C_{M}(x) \Varangle U(M)$. The product $n m$ is taken modulo $M$. (4) is called the conjugacy class of $\phi_{n}$ in $C_{M} \Varangle U(M)$.

\subsection{Character Extension}

Throughout this section $H$ is a normal subgroup of $G$ and $K$ is an arbitrary subgroup of $G$. Following Corollary 1.5, we assume $G=H \Varangle K$. The main results on character extensions will be proved under this assumption.

Suppose $\rho$ is a character of $K$. We say that $\rho$ extends to a character of $G$ if there exists a character $\gamma$ of $G$ such that $\rho$ is the restriction of $\gamma$ to $K$. Generally, even if $K$ is a normal subgroup of $G$, a character $\rho$ of 
$K$ will not necessarily extend to a character of $G$. This obstruction is a major difference between the abelian and the nonabelian group cases.

The following result establishes a necessary condition for a character $\tau$ of the normal subgroup $H$ of $G$ to have an extension to a character of $G$. For groups of the form $G=H \Varangle K$, it is also a sufficient condition.

THEOREM 33 If a character $\tau$ of $H$ extends to a character of $G$, then $G$ centralizes $\tau$.

THEOREM 34 If $\tau$ is a character of $H$ and $G_{1}$ is a subgroup of $G$ containing $H$ such that $\tau$ extends to a character of $G_{1}$, then $G_{1} \subset G(\tau)$.

As a special case, we have the following corollary.

COROLlary 1.5 If $\tau$ is a character of $H$ and $H=G(\tau)$, then $\tau$ cannot be extended to a character of any subgroup of $G$ strictly containing $H$.

In the language introduced below, if $\tau$ is a character of $H$ such that $H=G(\tau)$, then $\tau$ is a maximal character in $G$.

Assume $G=H \Varangle K$ for the rest of this chapter. A typical point $x \in G$ will be written uniquely as

$$
x=u v, \quad u \in H, v \in K,
$$

with the understanding that $u=u \cdot 1$ and $v=1 \cdot v$.

THEOREM 35 If $\tau$ is a character of $H$ centralized by $K$, then every product $\tau \lambda$, where $\lambda$ is a character of $K$, is a character of $G$ extending $\tau$.

Proof Choose any character $\lambda$ of $K$ and form the product $\rho=\tau \lambda$. We must show $\rho$ is a character of $G$. Since $v \tau=\tau v, v \in K$, we have

$$
x \rho=u v \tau \lambda=(u \tau)(v \lambda), \quad x=u v, u \in H, v \in K .
$$

By Theorem 30

$$
x \rho=\tau\left(u^{-1}\right) \lambda\left(v^{-1}\right) \tau \lambda=\rho\left(x^{-1}\right) \rho,
$$

and $\rho$ is an $L(G)$-eigen vector. Since $\rho(1)=1, \rho$ is a character of $G$, completing the proof.

Corollary 1.6 If $\tau$ is a character of $H$, then $\tau$ extends to a character of $G$ if and only if $K$ centralizes $\tau$. 
Theorem 35 describes a procedure for constructing characters of $G$ extending a character $\tau$ of $H$ centralized by $K$. In fact all the characters of $G$ extending $\tau$ are constructed in this way. For each character $\tau$ of $H$ centralized by $K$ denote by

$$
\operatorname{ext}_{\tau}(G)
$$

the collection of all characters of $G$ extending $\tau$.

THEOREM 36 If $\tau$ is a character of $H$ centralized by $K$, then $\operatorname{ext}_{\tau}(G)$ is the collection of all products $\tau \lambda$, where $\lambda$ is a character of $K$.

The collection $G^{*}$ of all characters of $G$ is the disjoint union of the sets

$$
\operatorname{ext}_{\tau}(G), \quad \tau \in H^{*} \text { and } \tau \text { centralized by } K \text {. }
$$

EXAMPLE 37 If $p$ is prime, there are $p-1$ characters of the group $C_{p}(x) \Varangle U(p)$. The only character of $C_{p}(x)$ which extends to $C_{p}(x) \Varangle U(p)$ is the trivial character $\phi_{0}$. Since $U(p)$ has order $p-1$, there are $p-1$ characters $\lambda_{j}, 0 \leq j<p-2$, of $U(p)$. The group algebra products

$$
\phi_{0} \lambda_{j}, \quad 0 \leq j<p-2,
$$

are the $p-1$ characters of $C(p) \Varangle U(p)$.

ExAmple 38 For $N \geq 2$, the characters of the normal subgroup

$$
C_{N}(x)=\left\{x^{m}: 0 \leq m<N\right\}
$$

of the dihedral group $\mathcal{D}_{2 N}=\mathcal{D}_{2 N}\left(x, k_{N-1}\right)$ are given by $\phi_{n}: C_{N}(x) \longrightarrow$ $U_{N}, 0 \leq n<N$,

$$
\phi_{n}\left(x^{m}\right)=v^{n m}, \quad 0 \leq m<N, v=e^{2 \pi i \frac{1}{N}} .
$$

Since $\mathcal{D}_{2 N}=C_{N}(x) \Varangle K$, where $K=\left\{1, k_{N-1}\right\}$ and

$$
k_{N-1} x k_{N-1}^{-1}=x^{-1} \text {, }
$$

$\phi_{n}$ is centralized by $K$ if and only if $v^{n}=e^{2 \pi i \frac{n}{N}}$ is real. For $N$ odd, we must have $n=0$ and the trivial character $\phi_{0}$ is the only character of $C_{N}(x)$ centralized by $K$. If $N$ is odd, $\mathcal{D}_{2 N}$ has two characters, the trivial character and the character $\rho_{1}$ defined by

$$
\rho_{1}\left(x^{m} k_{N-1}^{j}\right)=(-1)^{j}, \quad 0 \leq m<N, 0 \leq j<2 .
$$

For $N$ even, we must have $n=0$ or $n=\frac{N}{2}$ and the trivial character $\phi_{0}$ and the character $\phi_{\frac{N}{2}}$ are the only characters of $C_{N}(x)$ centralized by 
$K$. If $N$ is even, $\mathcal{D}_{2 N}$ has four characters, the trivial character $\rho_{0}$ and the three characters defined by

$$
\begin{array}{r}
\rho_{1}\left(x^{m} k_{N-1}^{j}\right)=(-1)^{j}, \rho_{2}\left(x^{m} k_{N-1}^{j}\right)=(-1)^{m}, \rho_{3}\left(x^{m} k_{N-1}^{j}\right)=(-1)^{m+j}, \\
0 \leq m<N, 0 \leq j<2 .
\end{array}
$$

\subsection{Maximal Extensions}

We continue to assume that $G=H \Varangle K$. If a character $\tau$ of $H$ is not centralized by $K$, then $\tau$ cannot be extended to a character of $G$. Since $H$ is a subgroup of $G(\tau), G(\tau)$ has the form

$$
G(\tau)=H \Varangle K(\tau),
$$

where $K(\tau)$ is the centralizer of $\tau$ in $K$,

$$
K(\tau)=\left\{v \in K: v \tau v^{-1}=\tau\right\} .
$$

THEOREM 39 If $\tau$ is a character of $H$, then $\tau$ extends to a character of $G(\tau)=H \Varangle K(\tau)$.

Denote by

$$
\operatorname{ext}_{\tau}(G(\tau))
$$

the collection of all characters of $G(\tau)$ extending $\tau$.

THEOREM 40 If $\tau$ is a character of $H$, then $\operatorname{ext}_{\tau}(G(\tau))$ is the collection of all products in $\mathbf{C} G$ of the form $\tau \lambda$, where $\lambda$ is a character of $K(\tau)$.

For future use we have the following corollary.

Corollary 1.7 If $\tau$ is a character of $H$, then

$$
\sum_{\rho \in \operatorname{ext}_{\tau}(G(\tau))} \rho=\tau \sum_{\lambda \in K(\tau)^{*}} \lambda
$$

Suppose $G_{1}$ is an arbitrary group and $K_{1}$ is a subgroup of $G_{1}$. A character $\gamma$ of $K_{1}$ is called a maximal character in $G_{1}$ if $\gamma$ has no extension to a character of a subgroup of $G_{1}$ strictly containing $K_{1}$. Any extension of a character $\gamma$ of $K_{1}$ to a maximal character in $G_{1}$ is called a maximal extension of $\gamma$ in $G_{1}$.

THEOREM 41 If $\tau$ is a character of $H$ then $\operatorname{ext}_{\tau}(G(\tau))$ is a collection of maximal extensions of $\tau$ in $G$.

We will show that the left ideal generated by a maximal character is irreducible. 
Suppose $\tau$ is a character of $H$ and $\rho$ is a character of $G(\tau)$ extending $\tau$. $G(\tau)$ is not necessarily a normal subgroup of $G$. Consider the centralizer $G(\rho)$ of $\rho$ in $G$. Since the support of $\rho$ is $G(\tau), G(\rho)$ normalizes $G(\tau)$,

$$
y z y^{-1} \in G(\tau), \quad z \in G(\tau), y \in G(\rho) .
$$

$G(\tau)$ centralizes $\rho$ implying $G(\tau)$ is a normal subgroup of $G(\rho)$.

THEOREM 42 If $\tau$ is a character of $H$ and $\rho$ is a character of $G(\tau)$ extending $\tau$, then $G(\rho)=G(\tau)$.

\subsection{Abelian by Abelian Semidirect Products}

Groups of the form $G=A \Varangle B$ with $A$ and $B$ abelian groups are perhaps the simplest generalizations of abelian groups. Not surprisingly, the DSP of this class of groups closely resembles that of abelian groups.

We proved that each character $\tau$ of $A$ extends to a character of the centralizer $G(\tau)=A \Varangle B(\tau)$ and that the set $\operatorname{ext}_{\tau}(G(\tau))$ of all characters of $G(\tau)$ extending $\tau$ consists of maximal characters in $G$.

\section{$3.12 E X T(G, A)$}

Suppose $\tau$ is a character of $A$. The centralizer of $\tau$ in $G$ is $G(\tau)=$ $A \Varangle B(\tau)$. Denote by

$$
\operatorname{ext}_{\tau}(G(\tau))
$$

the collection of all extensions of $\tau$ to characters of $G(\tau)$.

Denote by

$$
\operatorname{EXT}(G, A)
$$

the union of the sets $\operatorname{ext}_{\tau}(G(\tau))$ as $\tau$ runs over all characters of $A$. By Theorem 41 every character $\gamma$ in $\operatorname{ext}_{\tau}(G(\tau))$ is a maximal extension of $\tau$ and $\operatorname{EXT}(G, A)$ consists of maximal characters in $G$.

EXAMPLE 43 If $G=C_{p}(x) \Varangle U(p), p$ a prime, $\operatorname{EXT}\left(G, C_{p}(x)\right)$ has order $2(p-1)$. The characters $\phi_{n}, 0<n<p$, of $C_{p}(x)$ are maximal characters in $G$. The trivial character $\phi_{0}$ extends to $p-1$ characters of $G$.

Example 44 If $G=C_{8}(x) \Varangle U(8), E X T\left(G, C_{8}(x)\right)$ has order 16 . The characters $\phi_{1}, \phi_{3}, \phi_{5}$ and $\phi_{7}$ of $C_{8}(x)$ are maximal characters in $G$. As elements of $\mathbf{C} G$ they can be written explicitly as

$$
\left[\begin{array}{c}
\phi_{1} \\
\phi_{3} \\
\phi_{5} \\
\phi_{7}
\end{array}\right]=\left[\begin{array}{cccccccc}
1 & v & v^{2} & v^{3} & v^{4} & v^{5} & v^{6} & v^{7} \\
1 & v^{3} & v^{6} & v & v^{4} & v^{7} & v^{2} & v^{5} \\
1 & v^{5} & v^{2} & v^{7} & v^{4} & v & v^{6} & v^{3} \\
1 & v^{7} & v^{6} & v^{5} & v^{4} & v^{3} & v^{2} & v
\end{array}\right]\left[\begin{array}{c}
1 \\
x \\
\vdots \\
x^{7}
\end{array}\right]
$$


The character $\phi_{2}$ of $C_{8}(x)$ has two maximal extensions in $G$ to characters of $C_{8}(x) \Varangle K_{2}, K_{2}=\{1,5\}$. A similar remark can be made for the character $\phi_{6}$. The characters $\phi_{0}$ and $\phi_{4}$ of $C_{8}(x)$ extend to eight characters of $G$.

The first result extends, to the characters in $\operatorname{EXT}(G, A)$, the product formula of Theorem 9 for characters of abelian groups.

For $f \in \mathbf{C} G$ set $|f|$ equal to the order of the support of $f$.

Theorem 45 If $\gamma_{1}, \gamma_{2} \in \operatorname{EXT}(G, A)$, then

$$
\gamma_{1} \gamma_{2}= \begin{cases}\left|\gamma_{1}\right| \gamma_{1}, & \gamma_{1}=\gamma_{2} \\ 0, & \gamma_{1} \neq \gamma_{2}\end{cases}
$$

In the language of idempotent theory a nonzero element $e \in \mathbf{C} G$ is called an idempotent if $e^{2}=e$. Two idempotents $e_{1}$ and $e_{2}$ in $\mathbf{C} G$ are called orthogonal if $e_{1} e_{2}=e_{2} e_{1}=0$. Theorem 45 says that

$$
\left\{\frac{1}{|\gamma|} \gamma \in \operatorname{EXT}(G, A)\right\}
$$

is a set of pairwise orthogonal idempotents. Using the language of idempotents, we call $\operatorname{EXT}(G, A)$ a set of pairwise orthogonal characters in $G$.

The next result extends Theorem 11 to $\operatorname{EXT}(G, A)$.

THEOREM 46

$$
1=\sum_{\gamma \in E X T(G, A)} \frac{1}{|\gamma|} \gamma
$$

A set $\mathcal{I}$ of pairwise orthogonal idempotents is said to be complete if

$$
1=\sum_{e \in \mathcal{I}} e
$$

Theorems 45 and 46 say that the set

$$
\left\{\frac{1}{|\gamma|} \gamma: \gamma \in \operatorname{EXT}(G, A)\right\}
$$

is a complete set of pairwise orthogonal idempotents. Using the language of idempotents, we call $\operatorname{EXT}(G, A)$ a complete set of pairwise orthogonal characters in $G$.

Arguing as before, we have the following result.

THEOREM 47

$$
\mathbf{C} G=\bigoplus_{\gamma \in E X T(G, A)} \mathbf{C} G \gamma
$$




\subsection{A Basis of $\mathrm{CG} \gamma$}

Suppose $\gamma \in \operatorname{EXT}(G, A)$. We will show that $\mathbf{C} G \gamma$ can be multidimensional, determine the dimension of $\mathbf{C G \gamma}$ and construct a basis of $\mathbf{C} G \gamma$. In the next section we will show $\mathbf{C} G \gamma$ is irreducible.

Choose a complete system of left $G(\gamma)$-coset representatives in $G$,

$$
\left\{y_{s}: 1 \leq s \leq S\right\} \text {. }
$$

In general this system depends on $\gamma$. Since $G(\gamma)$ is a normal subgroup of $G$, by Theorem 32

$$
\gamma_{s}=y_{s} \gamma y_{s}^{-1}, \quad 1 \leq s \leq S,
$$

is the set of all conjugates of $\gamma$ in $G$ and

$$
\gamma_{s_{1}} \gamma_{s_{2}}=0, \quad 1 \leq s_{1}, s_{2} \leq S, s_{1} \neq s_{2} .
$$

Theorem 48 If $\gamma \in \operatorname{EXT}(G, A)$, then

$$
\left\{y_{s} \gamma: 1 \leq s \leq S\right\}
$$

is a basis of the space $\mathbf{C} G \gamma$.

Proof Suppose $x \in G$ and write $x=y_{s} z, 1 \leq s \leq S, z \in G(\gamma)$. By Theorem 30

$$
x \gamma=y_{s} z \gamma=\gamma\left(z^{-1}\right) y_{s} \gamma .
$$

Since the elements $x \gamma, x \in G$, span $\mathbf{C} G \gamma$, the elements $y_{s} \gamma, 1 \leq s \leq S$, span $\mathbf{C} G \gamma$.

To prove linear independence, suppose

$$
0=\sum_{s=1}^{S} \alpha(s) y_{s} \gamma, \quad \alpha(s) \in \mathbf{C} .
$$

For any $t, 1 \leq t \leq S$,

$$
0=\gamma_{t} 0=\sum_{s=1}^{S} \alpha(s) \gamma_{t} \gamma_{s} y_{s}=|\gamma| \alpha(t) y_{t} \gamma
$$

implying

$$
\alpha(t)=0, \quad 1 \leq t \leq S,
$$

completing the proof.

The basis of $\mathbf{C} G \gamma$

$$
\left\{y_{s} \gamma: 1 \leq s \leq S\right\}
$$

will be denoted by $B A S(\gamma)$. It consists of the left multiplications of $\gamma$ by the system (5). The basis of $\mathbf{C} G$ formed by the union of the sets $B A S(\gamma), \gamma \in \operatorname{EXT}(G, A)$, will be denoted by $B A S(G, A)$. 


\subsection{Irreducibility of $\mathrm{C} G \gamma$}

Theorem 49 If $\gamma \in \operatorname{EXT}(G, A)$, then $\mathbf{C} G \gamma$ is an irreducible left ideal.

Proof We continue to assume that $\left\{y_{s}: 1 \leq s \leq S\right\}$ is a complete system of left $G(\gamma)$-coset representatives in $G$. Suppose $V$ is a nonzero left ideal in $\mathbf{C} G \gamma$ and $f \in V, f \neq 0$. By Theorem 48

$$
f=\sum_{s=1}^{S} \alpha(s) y_{s} \gamma
$$

where $\alpha\left(s_{1}\right) \neq 0$ for some $1 \leq s_{1} \leq S$. Theorems 45 and 31 imply

$$
\gamma_{s_{1}} f=\sum_{s=1}^{S} \alpha(s) \gamma_{s_{1}} y_{s} \gamma=\sum_{s=1}^{S} \alpha(s) \gamma_{s_{1}} \gamma_{s} y_{s}=\alpha\left(s_{1}\right)|\gamma| \gamma_{s_{1}} y_{s_{1}} .
$$

Since $V$ is a left ideal and $f \in V, \gamma_{s_{1}} y_{s_{1}} \in V$ and

$$
\gamma=y_{s_{1}}^{-1} \gamma_{s_{1}} y_{s_{1}} \in V
$$

implying $V=\mathbf{C} G \gamma$, completing the proof.

\subsection{Expansion Coefficients}

A formula will be derived for the expansion coefficients of $f \in \mathbf{C} G$ relative to the basis $B A S(G, A)$. An algorithm implementing this formula will be constructed by making explicit the relationship between the characters of $A$ and $\operatorname{EXT}(G, A)$.

Suppose $\gamma \in \operatorname{EXT}(G, A)$. Choose a complete system of left $G(\gamma)$ coset representatives in $G$,

$$
y_{s}(\gamma), \quad 1 \leq s \leq S(\gamma)
$$

The dependence of the system on $\gamma$ is now explicitly expressed. The basis $B A S(G, A)$ of $\mathbf{C} G$ consists of all elements of the form

$$
y_{s}(\gamma) \gamma, \quad \gamma \in \operatorname{EXT}(G, A), 1 \leq s \leq S(\gamma) .
$$

The expansion of $f \in \mathbf{C} G$ relative to this basis will be written as

$$
f=\sum_{\gamma \in E X T(G, A)} \sum_{s=1}^{S(\gamma)} f_{\gamma}(s) y_{s}(\gamma) \gamma
$$

In this section we derive a formula for the expansion coefficients

$$
f_{\gamma}(s), \quad \gamma \in \operatorname{EXT}(G, A), 1 \leq s \leq S(\gamma)
$$


In the next section we will use this formula to derive a fast Fourier-like algorithm to compute these expansion coefficients.

By Theorem 45 if $f \in \mathbf{C} G$ and $\gamma \in \operatorname{EXT}(G, A)$, then

$$
f \gamma=|\gamma| \sum_{s=1}^{S(\gamma)} f_{\gamma}(s) y_{s}(\gamma) \gamma
$$

The expansion coefficients of the component of $f$ in $\mathbf{C} G \gamma$ relative to the basis $B A S(\gamma)$ are given by $f_{\gamma}(s), 1 \leq s \leq S(\gamma)$.

Theorem 50 If $f \in \mathbf{C} G$ and $\gamma \in \operatorname{EXT}(G, A)$, then

$$
f_{\gamma}(s)=\frac{1}{|\gamma|} \sum_{z \in G(\gamma)} f\left(y_{s}(\gamma) z\right) \gamma\left(z^{-1}\right), \quad 1 \leq s \leq S(\gamma) .
$$

Proof Set $y_{s}=y_{s}(\gamma)$ and $S=S(\gamma)$ throughout the proof. For $x \in G$, write $x=y_{s} z, 1 \leq s \leq S, z \in G(\gamma)$, and

$$
f=\sum_{x \in G} f(x) x=\sum_{s=1}^{S} \sum_{z \in G(\gamma)} f\left(y_{s} z\right) y_{s} z .
$$

Since by Theorem $30, y_{s} z \gamma=\gamma\left(z^{-1}\right) y_{s} \gamma$, we have

$$
f \gamma=\sum_{s=1}^{S}\left(\sum_{z \in G(\gamma)} f\left(y_{s} z\right) \gamma\left(z^{-1}\right)\right) y_{s} \gamma .
$$

Comparing with (6) completes the proof.

\section{Examples}

We will compute $\operatorname{EXT}(G, A)$ and $B A S(G, A)$ for the groups $G=$ $A \Varangle B$, where $A=C_{N}(x) \times C_{N}(y)$ and $B=C_{2}\left(k_{a}\right) \times C_{2}\left(k_{b}\right)$ with

$$
a=\left[\begin{array}{rr}
-1 & 0 \\
0 & -1
\end{array}\right], \quad b=\left[\begin{array}{ll}
0 & 1 \\
1 & 0
\end{array}\right] \text {. }
$$

The fixed points in $A$ under actions by the elements of $B$ depend on whether $N$ is even or odd.

Fixed points

\begin{tabular}{|l|l||l|}
\hline & $N$ even & $N$ odd \\
\hline$k_{a}$ & $(0,0),\left(\frac{N}{2}, 0\right),\left(0, \frac{N}{2}\right),\left(\frac{N}{2}, \frac{N}{2}\right)$ & $(0,0)$ \\
$k_{b}$ & $(0,0),(1,1), \ldots,(N-1, N-1)$ & $(0,0),(1,1), \ldots,(N-1, N-1)$ \\
$k_{a} k_{b}$ & $(0,0),(1, N-1), \ldots,(N-1,1)$ & $(0,0),(1, N-1), \ldots,(N-1,1)$ \\
\hline
\end{tabular}


Apart from the difference in the first row, it is important to note that for $N$ even, $\left(\frac{N}{2}, \frac{N}{2}\right)$ is a fixed point for $k_{b}$ and $k_{a} k_{b}$, but is not in the indexing set if $N$ is odd.

Centralizer in $B$

\begin{tabular}{|c|l||l|}
\hline \multirow{2}{*}{ Centralizer } & \multicolumn{2}{|c|}{ character index } \\
\cline { 2 - 3 } & $N$ even & $N$ odd \\
\hline$B$ & $(0,0),\left(\frac{N}{2}, \frac{N}{2}\right)$ & $(0,0)$ \\
$B_{1}$ & $\left(\frac{N}{2}, 0\right), \quad\left(0, \frac{N}{2}\right)$ & None \\
$B_{2}$ & $(l, l), 1 \leq l<N, l \neq \frac{N}{2}$ & $(l, l), 1 \leq l<N$ \\
$B_{3}$ & $(l, N-l), 1 \leq l<N, l \neq \frac{N}{2}$ & $(l, N-l), 1 \leq l<N$ \\
\hline
\end{tabular}

\section{1 $\operatorname{EXT}\left(G_{1}, A\right)$}

$\operatorname{Ext}\left(G_{1}, A\right)$ can be computed from the fixed points and centralizers and the characters of the subgroups of $B$. If the subgroup $C$ of $B$ is the centralizer of the point $(k, l)$, then the collection of products in $\mathbf{C} G_{1}$,

$$
\tau_{k, l} \lambda, \quad \lambda \in C^{*}
$$

is the collection of all characters in $\operatorname{EXT}\left(G_{1}, A\right)$ extending $\tau_{k, l}$.

The characters of $B$ are

$$
\begin{aligned}
& \lambda_{0,0}=1+k_{a}+k_{b}+k_{a} k_{b}, \quad \text { (trivial character). } \\
& \lambda_{0,1}=1+k_{a}-k_{b}-k_{a} k_{b}, \\
& \lambda_{1,0}=1-k_{a}+k_{b}-k_{a} k_{b}, \\
& \lambda_{1,1}=1-k_{a}-k_{b}+k_{a} k_{b} .
\end{aligned}
$$

The characters of the subgroups of $B$ are given by restriction.

Characters of subgroups of $B$.

\begin{tabular}{|c|l|}
\hline Subgroups & characters \\
\hline$B_{0}$ & 1 \\
$B_{1}$ & $1+k_{a}, 1-k_{a}$ \\
$B_{2}$ & $1+k_{b}, 1-k_{b}$ \\
$B_{3}$ & $1+k_{a} k_{b}, 1-k_{a} k_{b}$ \\
\hline
\end{tabular}

The following table describes $\operatorname{EXT}\left(G_{1}, A\right)$, for even $N$, by describing the subsets $\operatorname{ext}_{\tau}\left(G_{1}(\tau)\right)$ of characters in $\operatorname{EXT}\left(G_{1}, A\right)$ extending $\tau \in A^{*}$. 
$\operatorname{EXT}\left(G_{1}, A\right), N$ even.

\begin{tabular}{|l|l|l|}
\hline$\tau$ & $\operatorname{ext}_{\tau}\left(G_{1}(\tau)\right)$ & $G_{1}(\tau)$ \\
\hline$\tau_{0,0}, \tau_{\frac{N}{2}, \frac{N}{2}}$ & $\tau \lambda_{0,0}, \tau \lambda_{0,1}, \tau \lambda_{1,0}, \tau \lambda_{1,1}$ & $G_{1}$ \\
\hline$\tau_{0, \frac{N}{2}}, \tau_{\frac{N}{2}, 0}$ & $\tau\left(1+k_{a}\right), \tau\left(1-k_{a}\right)$ & $A \Varangle B_{1}$ \\
\hline$\tau_{l, l}$ & $\begin{array}{l}\tau\left(1+k_{b}\right), \tau\left(1-k_{b}\right) \\
1 \leq l<N, l \neq \frac{N}{2}\end{array}$ & $A \Varangle B_{2}$ \\
\hline$\tau_{l, N-l}$ & $\tau\left(1+k_{a} k_{b}\right), \tau\left(1-k_{a} k_{b}\right)$ & $A \Varangle B_{3}$ \\
& $1 \leq l<N, l \neq \frac{N}{2}$ & \\
\hline
\end{tabular}

Characters $\tau$ of $A$ not listed above have $B_{0}=\{1\}$ as their centralizer and are maximal characters in $\operatorname{EXT}\left(G_{1}, A\right)$.

$\operatorname{EXT}\left(G_{1}, A\right), N$ odd.

\begin{tabular}{|l|l|l|}
\hline$\tau$ & $\operatorname{ext}_{\tau}\left(G_{1}(\tau)\right)$ & $G_{1}(\tau)$ \\
\hline$\tau_{0,0}$ & $\tau_{0,0} \lambda_{0,0}, \tau_{0,0} \lambda_{0,1}, \tau_{0,0} \lambda_{1,0}, \tau_{0,0} \lambda_{1,1}$ & $G_{1}$ \\
$\tau_{l, l}$ & $\tau_{l, l}\left(1+k_{b}\right), \tau_{l, l}\left(1-k_{b}\right), \quad 1 \leq l<N$ & $A \Varangle B_{2}$ \\
$\tau_{l, N-l}$ & $\tau_{l, N-l}\left(1+k_{a} k_{b}\right), \tau_{l, N-l}\left(1-k_{a} k_{b}\right), \quad 1 \leq l<N$ & $A \Varangle B_{3}$ \\
\hline
\end{tabular}

Characters $\tau$ of $A$ not of the form given above are maximal characters in $\operatorname{EXT}\left(G_{1}, A\right)$.

The basis $B A S\left(G_{1}, A\right)$ is the disjoint union of the bases $B A S(\gamma)$ of the irreducible left ideals $\mathbf{C} G_{1} \gamma, \gamma \in \operatorname{EXT}\left(G_{1}, A\right) . B A S(\gamma)$ is the set given by the left multiplications of $\gamma$ by the elements in the complete system of left $B(\gamma)$-coset representatives in $B$.

We will define an ordering on $B A S\left(G_{1}, A\right)$ which is compatible with the direct sum decomposition of $\mathbf{C} G_{1}$ into the irreducible left ideals $\mathbf{C} G_{1} \gamma, \gamma \in \operatorname{EXT}\left(G_{1}, A\right)$. The elements in each basis $B A S(\gamma)$ of $\mathbf{C} G_{1} \gamma$ occur as contiguous elements.

$\operatorname{EXT}\left(G_{1}, A\right)$ consists of all elements of the form

$$
\gamma=\tau_{k, l} \lambda, \quad 0 \leq k, l<N, \lambda \in B\left(\tau_{k, l}\right)^{*} .
$$

Order the characters of $B$ as defined and order the characters of subgroups. Order $\operatorname{EXT}\left(G_{1}, A\right)$ by ordering $(k, l, \lambda)$, lexicographically. In this ordering $\lambda$ is the fastest running parameter, followed by $l$ and then by $k$.

The ordering in $\operatorname{EXT}\left(G_{1}, A\right)$ induces an ordering on the collection of all bases $B A S(\gamma), \gamma \in E X T\left(G_{1}, A\right)$. Ordering the elements in each of these bases, we have ordered $B A S\left(G_{1}, A\right)$ with the required compatibility condition with respect to the direct sum decomposition. 


$$
B A S(\gamma), N=3
$$

\begin{tabular}{|l|l|}
\hline$B(\gamma)$ & $B A S(\gamma)$ \\
\hline$B$ & $\tau_{0,0} \lambda_{0,0}, \tau_{0,0} \lambda_{0,1}, \tau_{0,0} \lambda_{1,0}, \tau_{0,0} \lambda_{1,1}$ \\
\hline$B_{1}$ & None \\
\hline$B_{2}$ & $\tau_{1,1}\left(1+k_{b}\right), k_{a} \tau_{1,1}\left(1+k_{b}\right), \tau_{1,1}\left(1-k_{b}\right), k_{a} \tau_{1,1}\left(1-k_{b}\right)$, \\
& $\tau_{2,2}\left(1+k_{b}\right), k_{a} \tau_{2,2}\left(1+k_{b}\right), \tau_{2,2}\left(1-k_{b}\right), k_{a} \tau_{2,2}\left(1-k_{b}\right)$, \\
\hline$B_{3}$ & $\tau_{1,2}\left(1+k_{a} k_{b}\right), k_{a} \tau_{1,2}\left(1+k_{a} k_{b}\right), \tau_{1,2}\left(1-k_{a} k_{b}\right), k_{a} \tau_{1,2}\left(1-k_{a} k_{b}\right)$, \\
& $\tau_{2,1}\left(1+k_{a} k_{b}\right), k_{a} \tau_{2,1}\left(1+k_{a} k_{b}\right), \tau_{2,1}\left(1-k_{a} k_{b}\right), k_{a} \tau_{2,1}\left(1-k_{a} k_{b}\right)$, \\
\hline$B_{0}$ & $\tau_{0,1}, k_{b} \tau_{0,1}, k_{a} \tau_{0,1}, k_{a} k_{b} \tau_{0,1}$ \\
& $\tau_{0,2}, k_{b} \tau_{0,2}, k_{a} \tau_{0,2}, k_{a} k_{b} \tau_{0,2}$ \\
& $\tau_{1,0}, k_{b} \tau_{1,0}, k_{a} \tau_{1,0}, k_{a} k_{b} \tau_{1,0}$ \\
& $\tau_{2,0}, k_{b} \tau_{2,0}, k_{a} \tau_{2,0}, k_{a} k_{b} \tau_{2,0}$ \\
\hline
\end{tabular}

Figure 8. Normalized basis of $\mathbf{C}\left(\left(C_{3} \times C_{3}\right) \Varangle\left(C_{2} \times C_{2}\right)\right)$

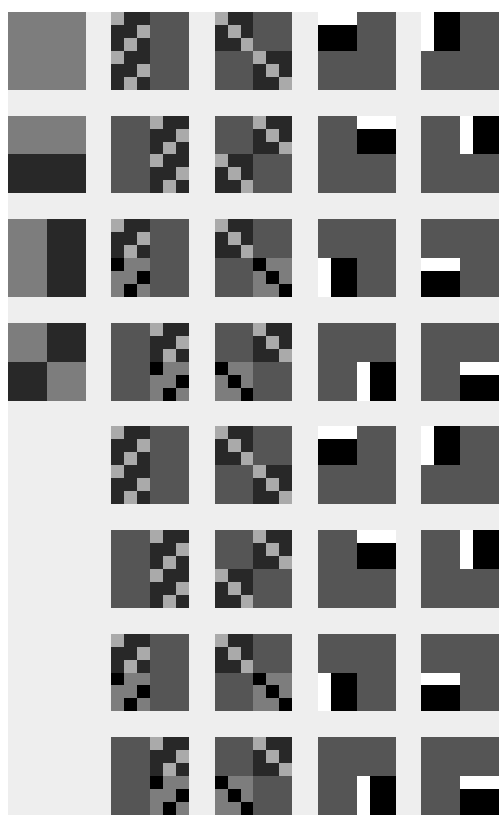

Real part

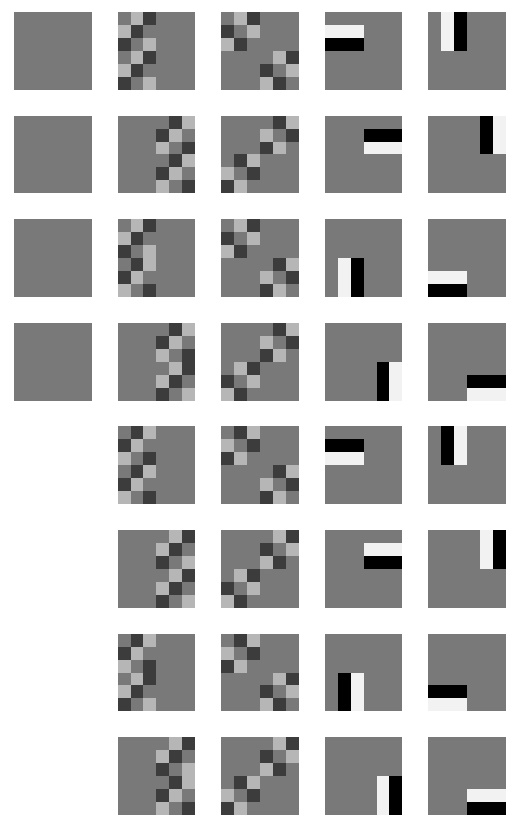

Imaginary part 
Figure 9. $\quad$ Normalized basis of $\mathbf{C}\left(\left(C_{4} \times C_{4}\right) \Varangle\left(C_{2} \times C_{2}\right)\right)$

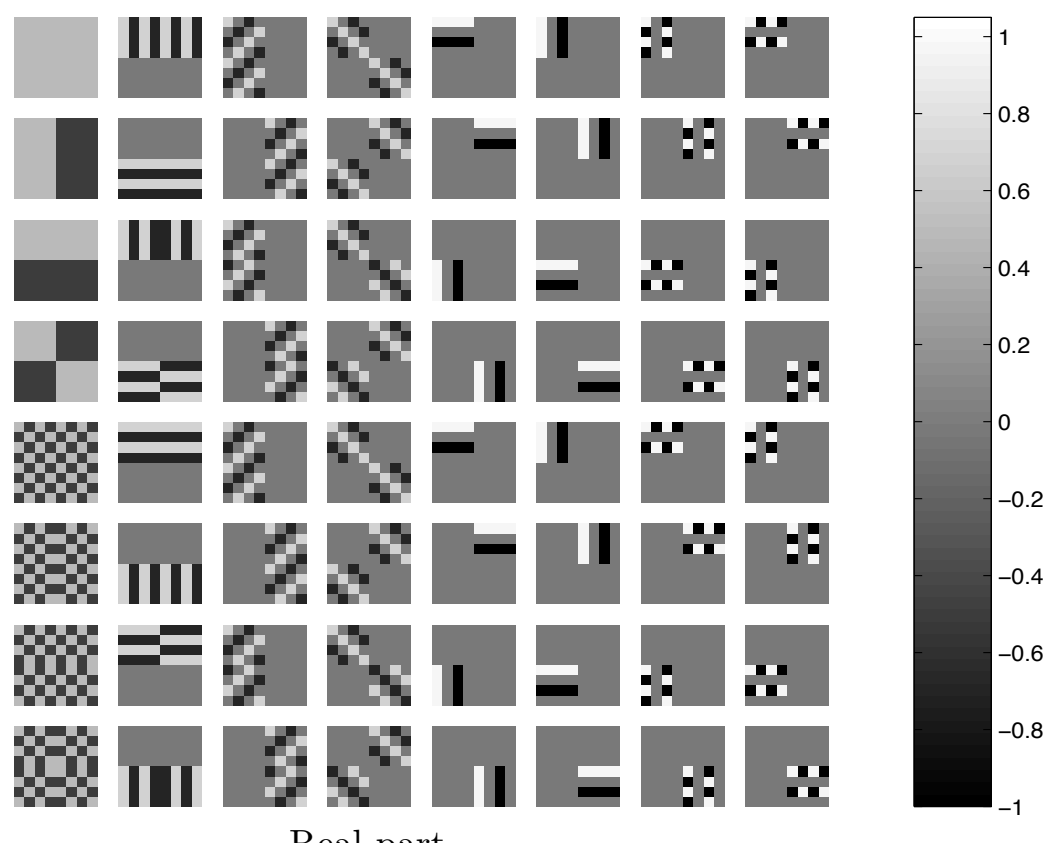

Real part

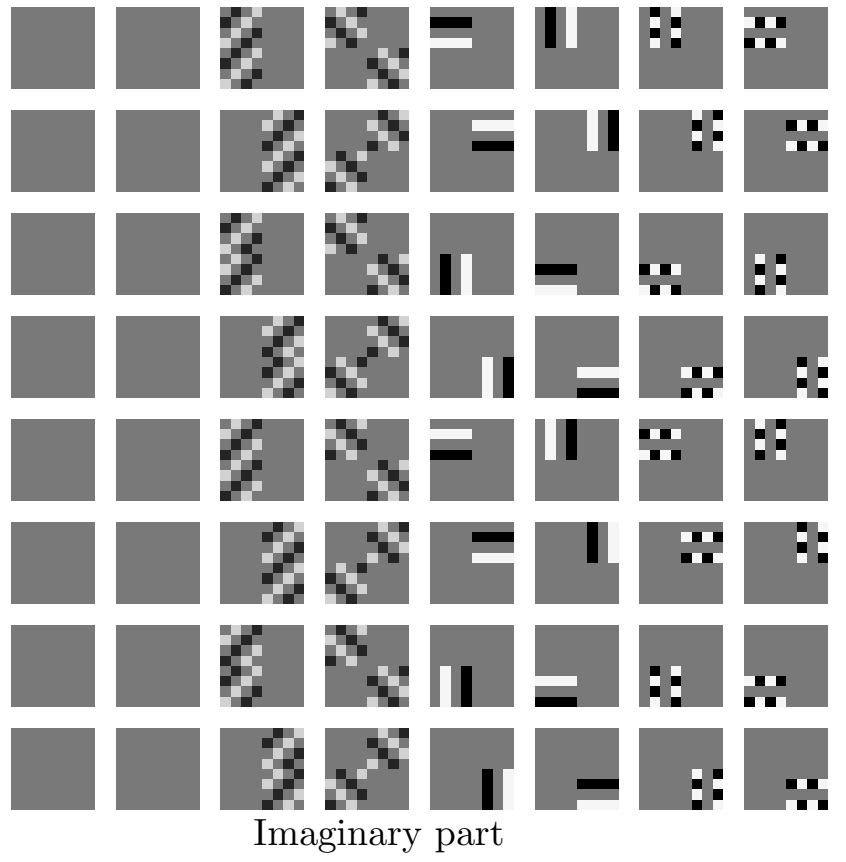


Figure 10. Generators of invariant subspaces of $\mathbf{C}\left(\left(C_{4} \times C_{4}\right) \Varangle\left(C_{2} \times C_{2}\right)\right)$

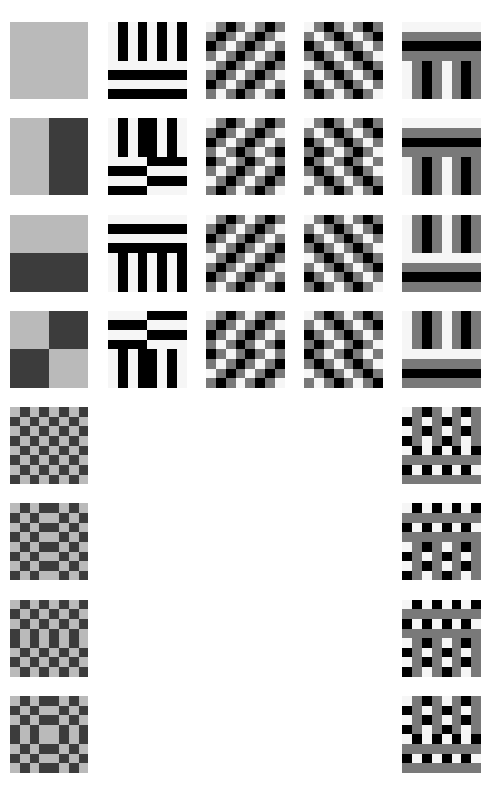

Real part

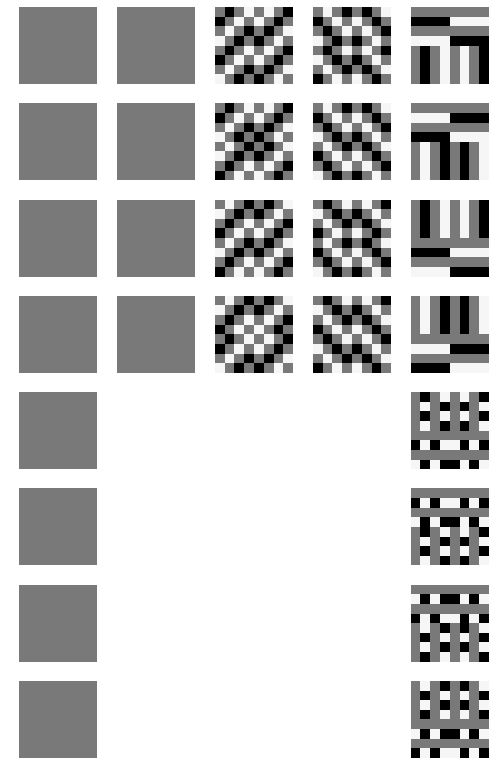

Imaginary part

In Figure 8, the coefficients of the basis of $G_{1}$, for $N=3$, are displayed as log-scaled intensity plots, with respect to this 2-dimensional indexing. The first column of 4 images corresponds to the characters of $G_{1}$, scaled by $\left|G_{1}\right|=36$. Each of these elements generates a 1-dimensional $\mathcal{L} G_{1}$-invariant subspace. The imaginary parts of the images in the first column are identically zero. The second column of 8 images, in pairs, corresponds to the basis of 2-dimensional invariant subspaces generated by the maximal extensions to $A \Varangle B_{2}$, scaled by 18 . The third column of 8 images, in pairs, corresponds to the basis of 2-dimensional invariant subspaces generated by the maximal extensions to $A \Varangle B_{3}$, scaled by 18. The remaining two columns of images, 4 images at a time, correspond to the basis of 4-dimensional invariant subspaces generated by the characters of $A$ which are maximal in $G_{1}$, scaled by 9 .

An index matrix of $G_{1}$, for $N=4$, is defined in an analogous way. In Figure 9, the coefficients of the basis of $G_{1}$, for $N=4$, are displayed as log-scaled intensity plots, with respect to this 2 -dimensional indexing. 
An intensity scale bar is appended on the right for reference, and applies to all image plots in this chapter.

The first column of 8 images corresponds to the characters of $G_{1}$, scaled by $\left|G_{1}\right|=64$. Each of these elements generates a 1-dimensional $\mathcal{L} G_{1}$-invariant subspace. The second column of 8 images, in pairs, corresponds to the basis of 2-dimensional invariant subspaces generated by maximal extensions to $A \Varangle B_{1}$, scaled by $\left|A \Varangle B_{1}\right|=32$. The imaginary parts of the images in the first two columns are identically zero. The third column of 8 images, in pairs, corresponds to the basis of 2dimensional invariant subspaces generated by the maximal extensions to $A \Varangle B_{2}$, scaled by 32 . The fourth column of 8 images, in pairs, corresponds to the basis of 2-dimensional invariant subspaces generated by the maximal extensions to $A \Varangle B_{3}$, scaled by 32 . The remaining columns of images, 4 images at a time, correspond to the basis of 4-dimensional invariant subspaces generated by the characters of $A$ which are maximal in $G_{1}$, scaled by 16 .

In Figure 10, the 28 generators of invariant subspaces are displayed. These are the results of adding the basis elements belonging to an invariant subspace.

\section{Group Transforms}

Suppose $G$ is an arbitrary group and $\Delta$ is a complete system of primitive, pairwise orthogonal idempotents in $\mathbf{C} G$. The $G$-group transform or simply $G$-transform is the collection of right multiplications of $\mathbf{C} G$

$$
\{R(e): e \in \Delta\} .
$$

Reference to $\Delta$ will always be suppressed. Each right multiplication in (7) is a $G$-component filter. Any $G$-component filter or sum of $G$-component filters is a $G$-filter.

The $G$-transform of $f \in \mathbf{C} G$ is the collection

$$
\{R(e) f: e \in \Delta\} .
$$

Each product in (8) is a $G$-spectral component of $f$. Any $G$-spectral component or sum of $G$-spectral components of $f$ is a $G$-filtering of $f$.

For an abelian group $A$ we always take $\Delta=\Delta(A)$. Abelian group transforms are probably well-known to the reader. Since each filter space of the $A$-transform

$$
\mathbf{C} A e, \quad e \in \Delta(A)
$$

is one-dimensional, the $A$-spectral component

$$
f e=\widehat{f}(\tau) e, \quad e=\frac{1}{N} \tau,
$$


can be represented by $\widehat{f}(\tau)$. The A-spectral image of $f$ is the collection

$$
\left\{\widehat{f}(\tau): \tau \in A^{*}\right\} .
$$

For any abelian by abelian semidirect product $G=A \Varangle B$ we always take $\Delta=\Delta(G)$. The filter spaces of the $G$-transform

$$
\text { CGe, } \quad e \in \Delta(G),
$$

are not necessarily one-dimensional. The $G$-spectral image of $f$ is the collection of expansion coefficients of $f$ relative to the basis $B A S(G, A)$.

A $G$-filter is a linear operator $P: \mathbf{C} G \longrightarrow \mathbf{C} G$ satisfying

$$
P(y f)=y P(f), \quad f \in \mathbf{C} G, y \in G .
$$

By linearity if $P$ is a $G$-filter, then

$$
P(g f)=g P(f), \quad f, g \in \mathbf{C} G .
$$

Every $f \in \mathbf{C} G$ defines a $G$-filter $R(f)$ by

$$
R(f) g=g f, \quad g \in \mathbf{C} G .
$$

$R(f)$ is called right multiplication by $f$. In general $L(f)$ is not a $G$-filter.

TheOREm 51 If $P$ is a G-filter, then there exists $f \in \mathbf{C} G$ such that $P=R(f)$.

The image of the $G$-filter $R(f)$ is the left ideal $\mathbf{C} G f$. We call $\mathbf{C} G f$ the filter space of $R(f)$. Since we can have $\mathbf{C} G f_{1}=\mathbf{C} G f_{2}$, for distinct $f_{1}, f_{2} \in \mathbf{C} G$, distinct $G$-filters can have the same filter space.

\subsection{Matched Filtering}

Suppose $G$ is an arbitrary group and $\Delta$ is a complete system of primitive, pairwise orthogonal idempotents in $\mathbf{C} G$. For $f \in \mathbf{C} G$, the $G$ matched filter of $f$ is the $G$-filter

$$
e_{f}=\sum_{f e \neq 0} e .
$$

The summation in (9) runs over all idempotents $e \in \Delta$ such that $f e \neq 0$. The $G$-matched filter of $f$ satisfies

$$
f e_{f}=f
$$


In a typical image processing application an image $f \in \mathbf{C} G$ is contained in noise and background. The problem is to separate the target $f$ from the noise and background. The image data has the form

$$
g=f+\eta,
$$

where $\eta \in \mathbf{C} G$ represents the noise and background. The $G$-matched filtering

$$
g e_{f}=f+\eta e_{f}
$$

is usually the optimal $G$-filtering for separating $f$ from $\eta$. Examples of $G$-matched filtering will be given in the following sections.

The concept of a $G$-matched filter is a major tool in both abelian and nonabelian group filter design. As defined, the formal structure of a $G$-matched filter does not change with different choices of group $G$. However, the choice of indexing group is a critical parameter affecting several important characteristics of the resulting matched filters. We have distinguished two properties which can vary as the indexing group varies: invariance under left group actions and the extent and form of image domain locality. The first will be discussed in this section. Image domain locality is the topic addressed in the next section.

For an image $f \in \mathbf{C} G$, the $G$-equivalence class of images containing $f$ is the equivalence class of images in the set

$$
\{x f: x \in G\} \text {. }
$$

The $G$-equivalence class containing $f$ depends on the actions of the left multiplications from $G$ on $f$ and can vary as $G$ varies.

The $G$-matched filter of $f$ satisfies

$$
x f e_{f}=x f, \quad x \in G .
$$

This implies that the $G$-matched filter of $f$ is the $G$-matched filter of every image $G$-equivalent to $f$. In applications this means that the $G$ matched filter constructed for an image $f$ will be the optimal filtering not only for $f$, but also for all $G$-equivalent images.

\subsection{Image Domain Locality}

Image domain locality is a key component of nonabelian group filters not available with abelian group filters. To understand where this property comes from and how it can be controlled at the group level, we will show that the expansion coefficients in nonabelian group DSP can encode local image space information.

The explanation is related to the character extension problem. If $A$ is an abelian group, then this is straightforward. The basis for Fourier 
representation is the collection of characters. Relative to this basis, each expansion coefficient contains image information across the image plane. These coefficients are sensitive to all local changes. The placing of a geometric structure in an image data set affects all expansion coefficients.

The character extension problem is more complicated for an abelian by abelian semidirect product $G=A \Varangle B$. As we have pointed out there exist characters of $A$, which can not be extended to characters of $G$. The characters defining $\Delta(G)$ are maximal characters in $G$, but some have proper subgroups of $G$ as their supports.

Suppose $e \in \Delta(G)$ and

$$
e=\frac{1}{|\gamma|} \gamma
$$

where $\gamma$ is a character of the subgroup $G(\gamma)$ of $G$. For the discussion assume $G(\gamma)$ is a proper subgroup of $G$. If

$$
\left\{y_{s}: 1 \leq s \leq S\right\}
$$

is a complete system of left $G(\gamma)$-coset representatives in $G$, then

$$
\left\{y_{s} e: 1 \leq s \leq S\right\}
$$

is a basis for the irreducible left ideal $\mathbf{C} G e$. The key point is that $G(\gamma)$ is a proper subgroup of $G$ and the basis (10) is supported on pairwise disjoint left-cosets. For each $s, 1 \leq s \leq S$, the basis element $y_{s} e$ is localized in the image domain to the left coset $y_{s} G(\gamma)$.

Suppose $f \in \mathbf{C} G$. We can write

$$
f e=\sum_{s=1}^{S} \alpha(s) y_{s} e .
$$

If $g \in \mathbf{C} G$ is supported in $y_{t} G(\gamma)$ for some $1 \leq t \leq S$, then

$$
(f+g) e=\sum_{s \neq t} \alpha(s) y_{s} e+\alpha^{\prime}(t) y_{t} e .
$$

The effect of the local image domain change on the expansion coefficients of the filtered image, resulting from the addition of $g$, is to change exactly one coefficient. The sensitivity to the local image domain depends on the relative orders of $G$ and $G(\gamma)$.

For a fixed character $\tau$ of $A$, the left ideal $\mathbf{C} G \tau$ can be written as

$$
\mathbf{C} G \tau=\bigoplus_{\lambda \in B(\tau)^{*}} \mathbf{C} G \tau \lambda
$$


$\mathbf{C} G \tau$ is not necessarily irreducible. We can write

$$
f \tau=\frac{1}{|B(\tau)|} \sum_{\lambda \in B(\tau)^{*}} \sum_{s=1}^{S} \alpha_{\lambda}(s) y_{s} \tau \lambda .
$$

This expansion shifts the basis in both the image and spectral image domains and can be viewed as a localized image-spectral image domain expansion in analogy to time-frequency expansions. Local image domain changes affect a set of prescribed coefficients and these changes describe the local image domain change in both image and spectral image domains.

\section{Group Filters}

In this section we study and compare properties of abelian and nonabelian group filters by illustrating their effects on lines and line segments. These examples will concentrate on the image domain locality and the left group multiplication invariance of the filters.

\subsection{Abelian group filtering}

For $N=64$, set

$$
A_{0}=\sum_{k=0}^{N-1} \tau_{0, k} \in \mathbf{C} A .
$$

$A_{0}$ is the matched filter of the vertical line and all its abelian group translates.

The following examples show detection and location by $A_{0}$ of a vertical line and several of its abelian group translates. Varying levels of noise are added to generate the images in Figure 12. The noise is modeled as Gaussian with zero-mean. Levels of noise are indicated by the standard deviation (sd). Images in Figure 13 are the results of filtering by $A_{0}$. Even in severe noise, the location of maximum intensity yields the positions of the translates of a vertical line.

Figure 11. Images containing a vertical line

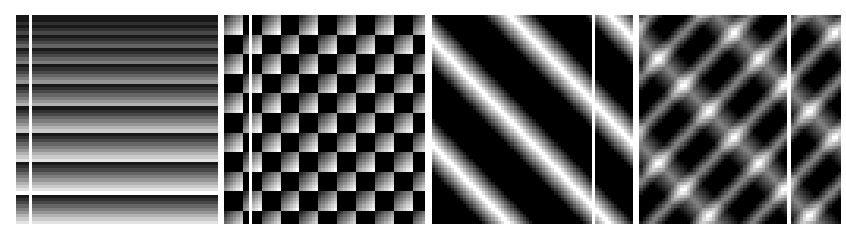


Figure 12. Noisy images containing a vertical line

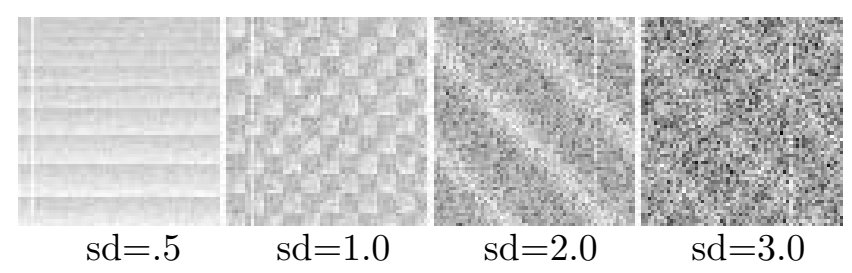

Figure 13. Results of Filtering by $A_{0}$

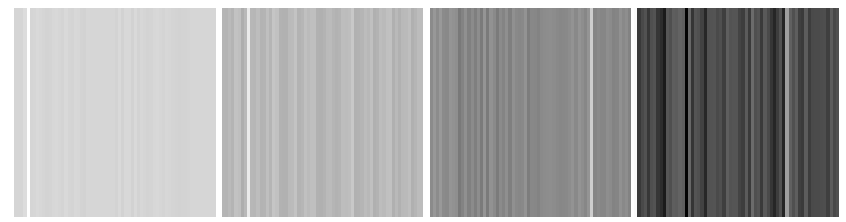

In Figures 14 and 15 the translates of a vertical line are replaced by line segments of one half the length. The placement of the vertical line segment is given by the coordinate of the starting position.

Figure 14. Images containing a vertical line segment

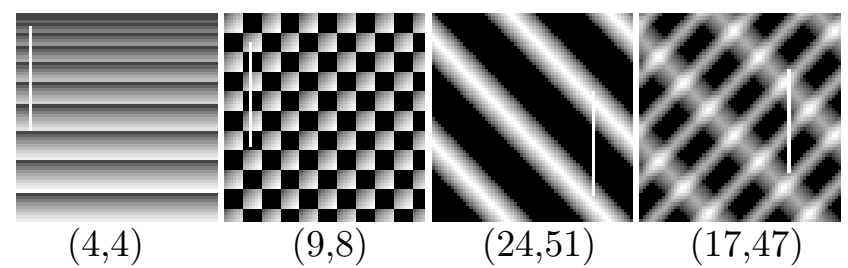

Figure 15. Noisy images containing a vertical line segment

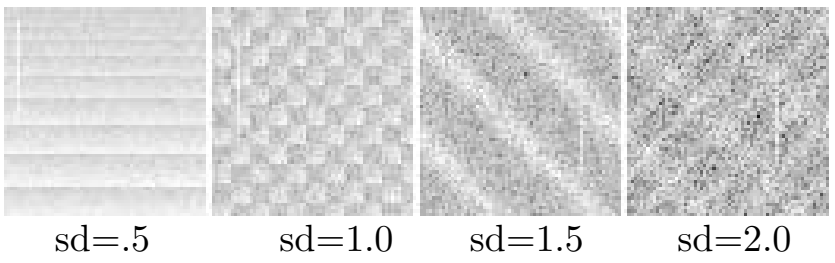

The last example shows that as the length of the line diminishes, the performance of the filter $A_{0}$ for detecting line segments worsens. 
Figure 16. Results of Filtering by $A_{0}$

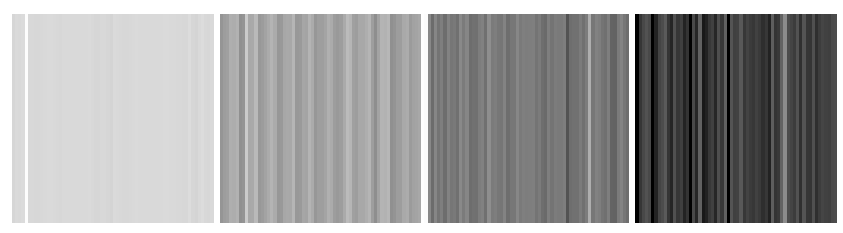

Figure 17. Images of vertical line segments

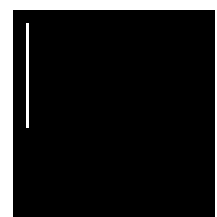

$(4,4)$

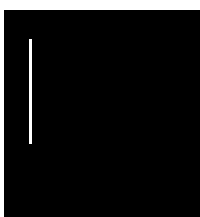

$(9,8)$

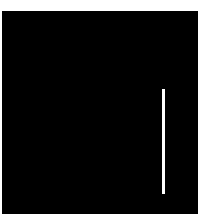

$(24,51)$

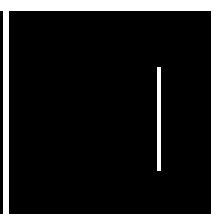

$(17,47)$

Figure 18. Results of Filtering by $A_{0}$
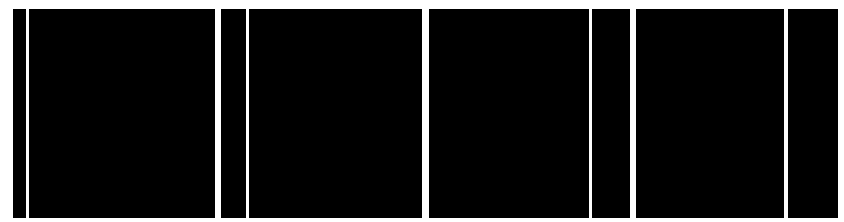

Moreover, the vertical position of the line segment cannot be detected, even without any noise as shown in Figure 18.

The preceding examples show one of the major deficiencies of abelian group filtering, the lack of image domain locality.

\subsection{Nonabelian group filtering}

Consider the group

$$
G_{4}=A \Varangle C_{2}\left(k_{c}\right)
$$

and the group filter defined by

$$
P_{0}=\frac{1}{N} \sum_{k=1}^{N-1} \tau_{0, k}+k_{c} \tau_{0, k} .
$$

The choice of $P_{0}$ was made for its simplicity, and because it is symmetric or real valued. An intensity plot of the filter is shown below for $N=32$ along with the intensity color scheme. 
Figure 19. Intensity plot of the filter $P_{0}, N=32$.

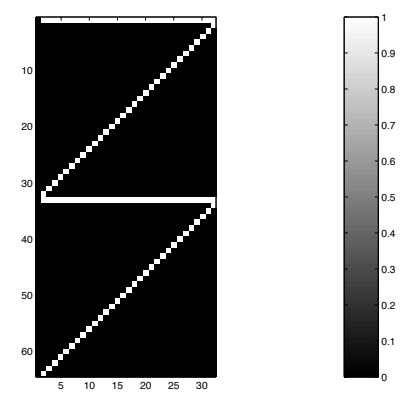

The following example shows the importance of matching the filter to the targeted image. The first test image contains a vertical line segment, while the second test image contains a line segment of slope 1. In Figure 20 , the first and third images contain a vertical line segment, while the second and fourth images contain a line segment of slope 1. Noise levels are indicated by the standard deviation $(s d)$. Figure 21 displays the results of applying $P_{0}$.

Figure 20. Test images in noise

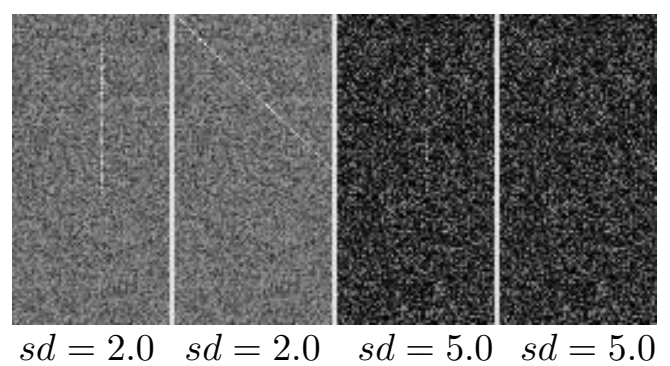

The filter $P_{0}$ is matched to the vertical line and to all left multiplications of the vertical line by the elements of $G_{4}$. Since the line segment of slope 1 is not contained in the collection of $G_{4}$-equivalent images, it cannot be detected by $P_{0}$.

Invariance of $P_{0}$ with respect to left multiplication by the elements from $G_{4}$ implies that these left actions do not affect filter performance. The previous figure shows this for left multiplications by $x^{m} y^{n}, 0 \leq m$, $n<N$. 
Figure 21. Results of applying $P_{0}$

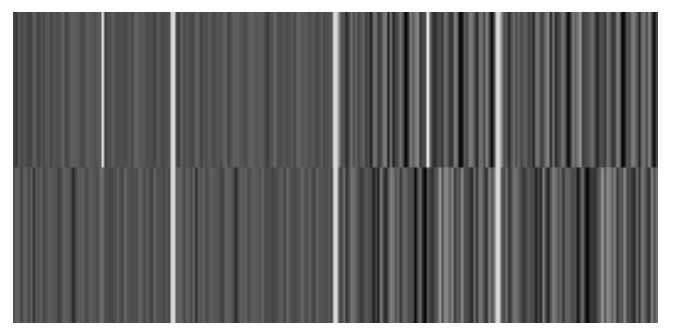

Figure 22. $\quad k_{c}$-translates of the images in Figure 20
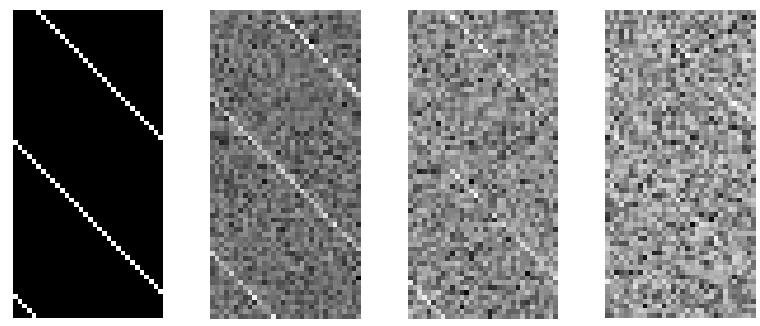

Figure 23. Results of filtering by $P_{0}$
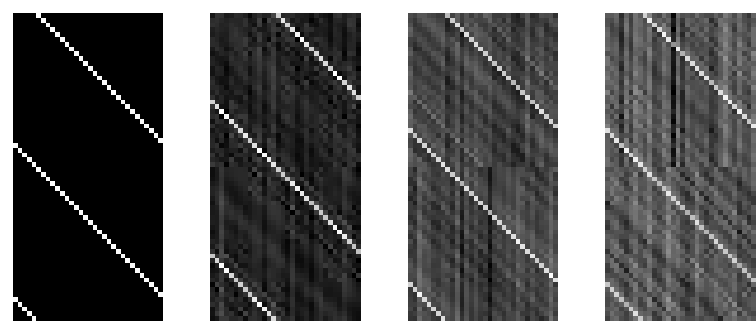

Each frame in Figure 22 displays the $k_{c}$-translate of the corresponding frame in Figure 20. Figure 23 displays the results of filtering the images in Figure 22 by $P_{0}$.

The next pair of figures show the implications of invariance and image domain locality. In Figure 24 a vertical line segment of length 32 is placed arbitrarily, and the horizontal position is recorded. The problem is to determine the position using the filter $P_{0}$. Figure 25 shows the results of this filtering by $P_{0}$. 
Figure 24. $\quad x^{m}$-translates of an image containing line segment of length 32

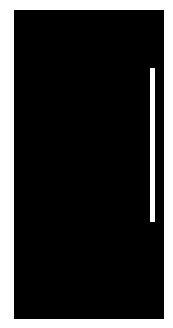

$m=11$

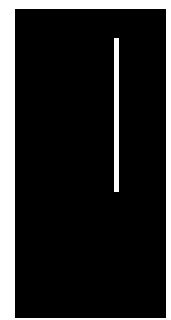

$m=5$

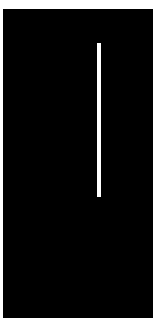

$m=6$

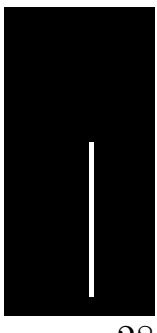

$m=28$

Figure 25. Results of filtering by $P_{0}$
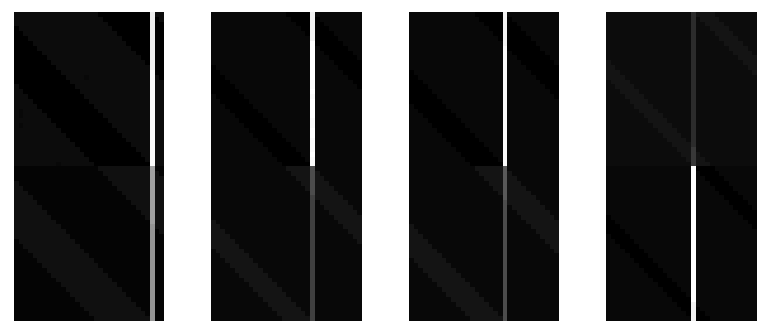

$(u, l)=(.585, .382)$

$(.761, .207)(.732, .236)$

$(.117, .851)$

The pair of numbers $(u, l)$ in Figure 23 are the intensities of the upper and lower half of the line segments. Note the close relationship between the intensities of the line segment and its position. (The intensity of the input line segment in Figure 25 is 1.0, but this information is only relative.) The relationship between the difference of intensities and position is given by

$$
m \sim N-\frac{u N}{u+l}
$$

Accuracy of the relationship (11) depends on $N$, up to $\frac{N}{16}$ pixels. Thus the smaller $N$ is the more accurate the relationship (11).

The same experiment is repeated with varying levels of noise. The position of the line segment is estimated from the filtered image using (11), and denoted by $m^{\prime}$ in Figure 27.

In the presence of noise, the intensity is not uniform through the upper or lower half of the line segment, but the variance is very small. Estimate of location in (11) is derived using the intensity at position 
Figure 26. $\quad x^{m}$-translates of an image containing line segment of length 32

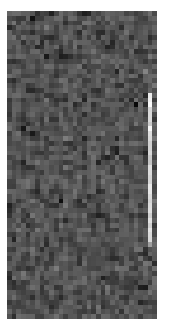

$s d=0.5$

$m=16$

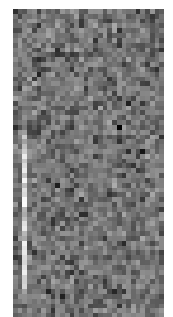

$s d=1.0$

$m=25$

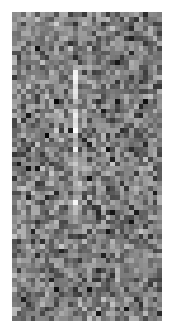

$s d=1.5$

$m=10$

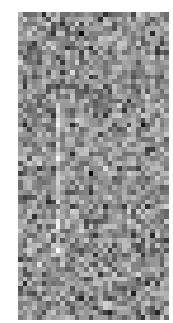

$s d=2.0$

$m=18$

Figure 2\%. Results of filtering by $P_{0}$

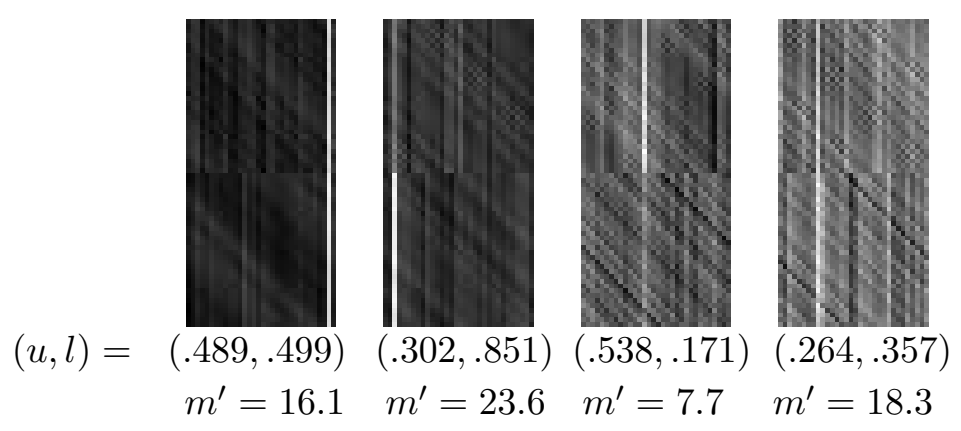

$(0, y)$ and $(N, y)$, where $y$ is the horizontal position of the line segment. A better estimate than (11) can be derived, especially in cases where noise characteristics are known.

As these experiments show, detection and location of vertical lines of uniform intensity of known lengths can be implemented using $P_{0}$. Using similar analysis, a relationship can be derived and the same implementation can be used to locate diagonal lines shown in Figure 22. Since $k_{c} x^{m} y^{n} k_{c}^{-1}=x^{m} y^{m-n}$, the $x$-intercept is equivalent to the horizontal location of the vertical line.

Note that the length of a line segment must be at least $N$ for exploiting the localization property consistently. More specifically, if the length of the line segment is less than $N$, in the case where the line segment lies entirely in lower or the upper of the two planes, we can localize the line segment only up to one of the two planes. This provides one major parameter determining the design of groups and filters in detection/localization applications: For consistent detection/localization 
Figure 28. Intensity plot of the filter $P_{1}, N=32$.

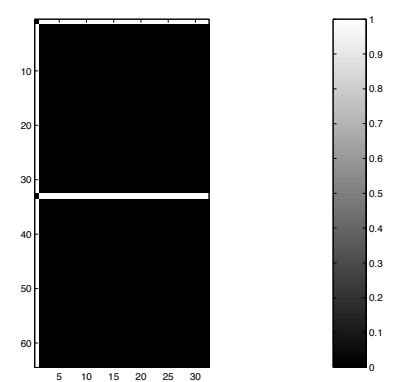

of, for example, a vertical line segment of length $N$ in an image of size $K_{1} N \times K_{2} N$, the following two approaches can be used.

1 Starting with a group $G=\left(C_{N} \times C_{N}\right) \Varangle C_{2}$, we can apply $P_{0}$ to subimages of size $2 N \times N$, overlapped by at least $N$.

2 Design filters associated to a group of the form $G=\left(C_{N} \times C_{N}\right) \Varangle$ $\left(C_{K_{1}} \times C_{K_{2}}\right)$. (Groups of the form $\left(C_{N} \Varangle C_{K_{1}}\right) \times\left(C_{N} \times C_{K_{2}}\right)$ will work as well for detection/localization of vertical or horizontal lines, but groups of the form $\left(C_{N} \times C_{N}\right) \Varangle\left(C_{K_{1}} \times C_{K_{2}}\right)$ provide more flexibility in shapes.)

The filter performance degrades as the noise level increases. This is particularly true with no prior knowledge of the noise characteristics. One method that will improve filter performance is to use a similar filter based on a different group of the same size. Consider the group

$$
G=\left(C_{N}(x) \times C_{N}(y)\right) \Varangle C_{2}\left(k_{e}\right),
$$

where $e=\left[\begin{array}{ll}0 & 1 \\ 1 & 0\end{array}\right]$. Construct the $G$-filter

$$
P_{1}=\frac{1}{N} \sum_{n=1}^{N-1} \tau_{0, k}+k_{e} \tau_{0, k} .
$$

$P_{1}$ is also real-valued. An intensity plot of $P_{1}$ is shown in Figure 28 .

In Figures $29-31$, results of applying $P_{0}$ and $P_{1}$ are shown in the third and fourth frames along with estimates of the location of the line segment. The first frame displays the line segment and the second frame displays the line segment embedded in background and noise. 
Figure 29. Results of applying $P_{0}$ and $P_{1}$

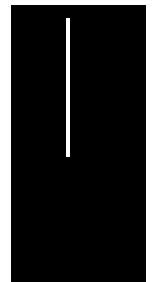

$m=3$

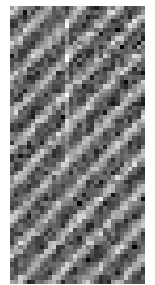

$s d=2.0$

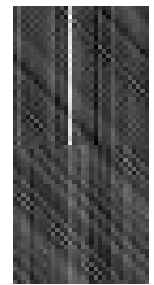

$m^{\prime}=5.0 \quad m^{\prime \prime}=5.6$

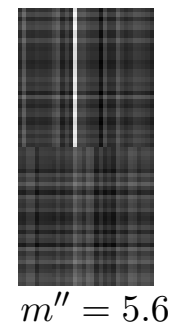

Figure 30. Results of applying $P_{0}$ and $P_{1}$
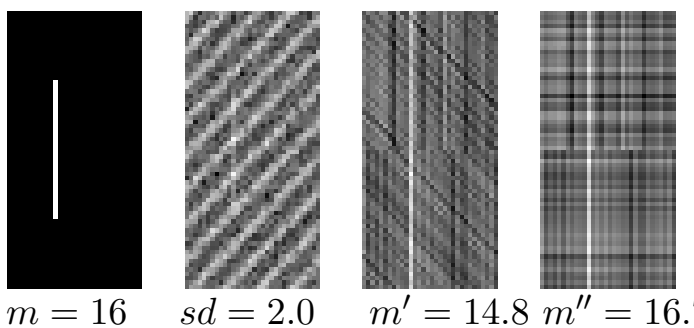

$m=16$

$s d=2.0$

Figure 31. Results of applying $P_{0}$ and $P_{1}$

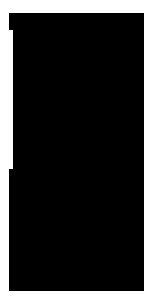

$m=4$

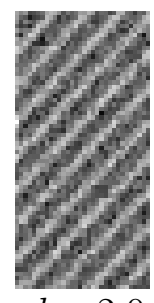

$s d=2.0$

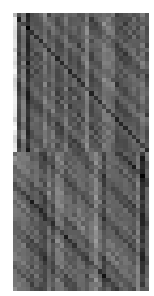

$m^{\prime}=5.5 \quad m^{\prime \prime}=2.2$

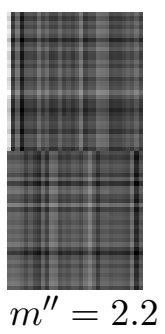

The accuracy of the estimate (11) differs between the filters $P_{0}$ and $P_{1}$ as the noise is filtered out in two distinct ways by the distinct convolutions defined by the two groups. This is another parameter determining design of a detection/location application. By applying several distinct groups, detection and location can be made more accurate. A trade-off is clearly the cost of processing. 


\section{Line-like Images}

In this section the performance of groups filters on non-digital, linelike objects is illustrated. Generally, filter performances are better due to the fact that non-digital lines have more than one pixel width.

In Figure 32 the first image is the digital line of slope 0 . The second image is generated by rotating the horizontal line by $27^{\circ}$ clockwise about the center of the image. Rotation is implemented using bilinear interpolation. The third image displays a portion of the rotated line near the center of rotation.

Figure 32. Result of rotating a line

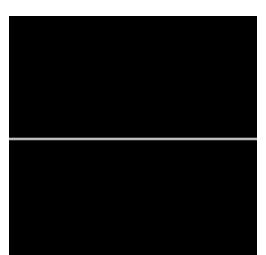

horizontal line through $(64,64)$

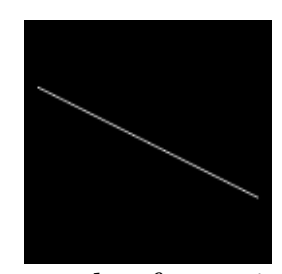

result of rotating the horizontal line

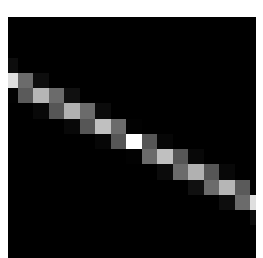

detail around center of rotation

The following example compares the results of filtering a digital test image and a rotated test image in varying levels of noise.

Figure 33. Test images in noise, $s d=.5$

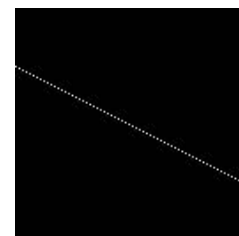

(a1)

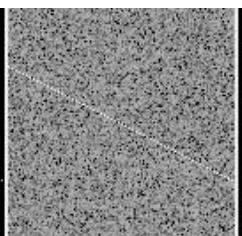

(b1)

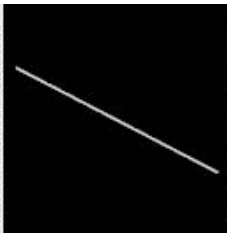

$(\mathrm{c} 1)$

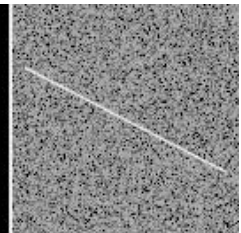

$(\mathrm{d} 1)$

Images in Figure 33 are as follows.
(a1) digital line segment of slope $-\frac{1}{2}$,
(c1) rotated line,
(b1) digital line in noise,
(d1) rotated line in noise

The next example illustrates the performance of matched filters in detecting and locating a line-like object in recorded data. 
Figure 34. Results of filtering in noise, $s d=.5$

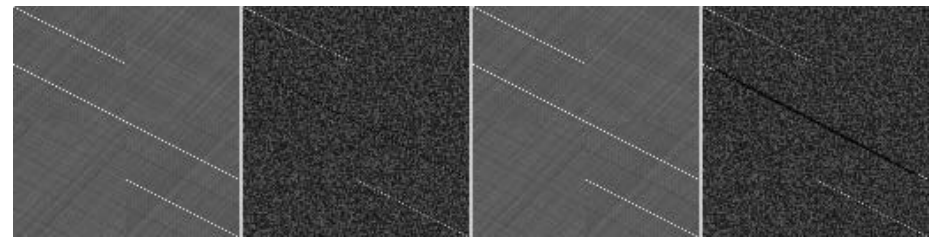

Figure 35. Sonar image of size $128 \times 128$ and result of filtering

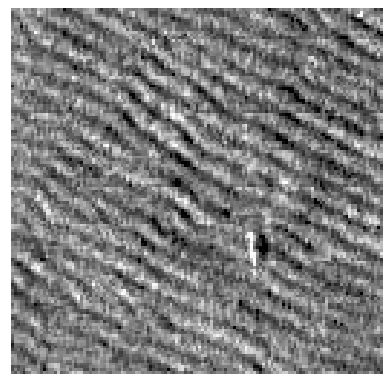

piece of data containing an object of interest

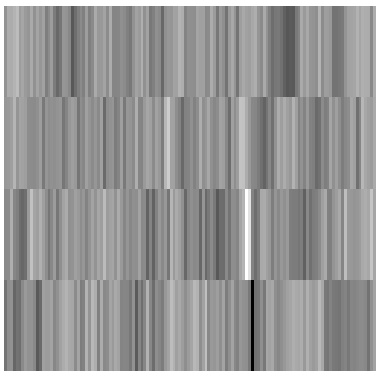

result of filtering

The next collection of examples are included to illustrate:

1 the diversity of images which can be created by composition of parallel lines of varying orientations,

2 the sensitivity of the matched filters to changes in orientations,

3 design strategy for matched filters whose properties vary with image size.

For a positive integer $K$ and $N=16 K$, consider the groups

$$
G_{6}=A \Varangle C_{4}\left(k_{a}\right), \quad G_{7}=A \Varangle C_{4}\left(k_{b}\right),
$$

where $A=C_{N}(x) \times C_{N}(y)$ and

$$
a=\left[\begin{array}{cc}
\frac{N}{2} & \frac{N}{4}+1 \\
\frac{N}{4}+1 & 0
\end{array}\right], \quad b=\left[\begin{array}{cc}
\frac{N}{2} & \frac{N}{4}-1 \\
\frac{N}{4}-1 & 0
\end{array}\right] .
$$


The sum of 1-dimensional idempotents in $\mathbf{C} G_{6}$ and in $\mathbf{C} G_{7}$ define matched filters

$$
\begin{aligned}
P_{6} & =\sum_{k=0}^{N-1} \sum_{j=0}^{3} \tau_{k,(M+1) k} \lambda_{j} \in \mathbf{C} G_{6}, \\
P_{7} & =\sum_{k=0}^{N-1} \sum_{j=0}^{3} \tau_{k,(M-1) k} \mu_{j} \in \mathbf{C} G_{7} .
\end{aligned}
$$

Figures 36 and 37 display the filters $P_{6}$ and $P_{7}$. Unlike the matched filters we have previously described, properties of $P_{6}$ and $P_{7}$ depend on $N$ which determines the image size.

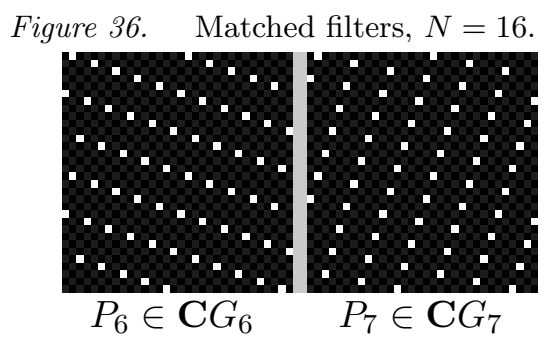

The first image in Figure 38 is a composition of two sets of intensities along parallel lines of slopes 3 and 4 . The results of applying $P_{6}$ and $P_{7}$ in noise are displayed. Noise is simulated as Gaussian of zero mean with varying values of standard deviation.

The last image in Figure 38 is not an algebraic object in that it belongs neither to $\mathbf{C} G_{6}$ nor $\mathbf{C} G_{7}$. It is simply the sum of two intensities defined on an array of size $2 N \times 2 N$.

In Figure 40, $\mathbf{g}$ is viewed as an element of $\mathbf{C} G_{7}$. The last image is again simply the sum of two intensities.

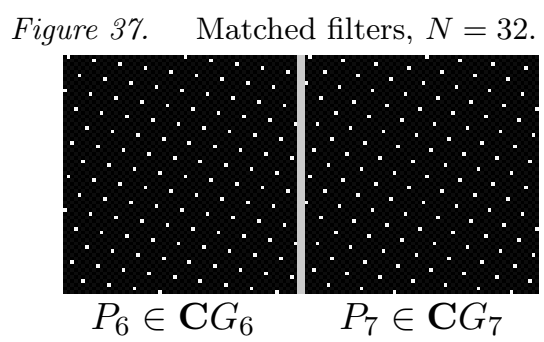


Figure 38. Results of filtering in noise

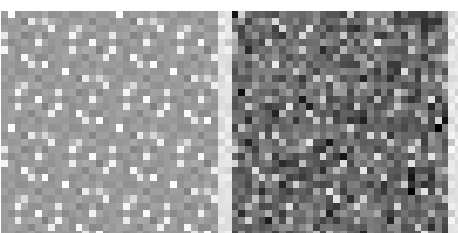

test image $\mathbf{f} \quad \mathbf{f}$ in noise

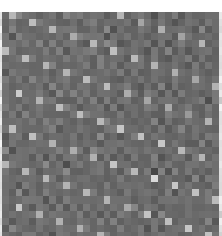

$P_{6}$

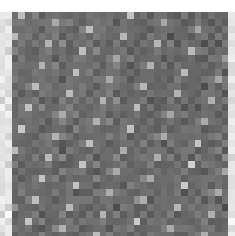

$P_{7}$

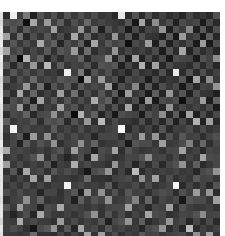

$P_{6}+P_{7}$

Figure 39. Results of filtering in noise
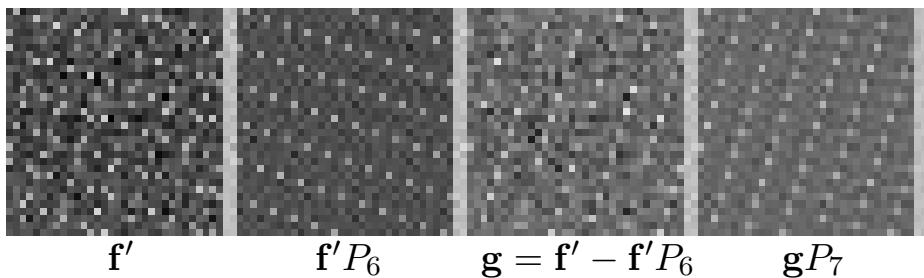

$\mathbf{g}=\mathbf{f}^{\prime}-\mathbf{f}^{\prime} P_{6}$

g $P_{7}$

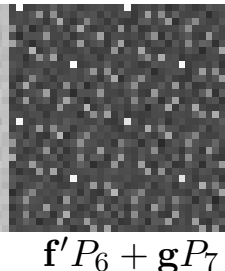

Figure 40. Results of filtering in noise
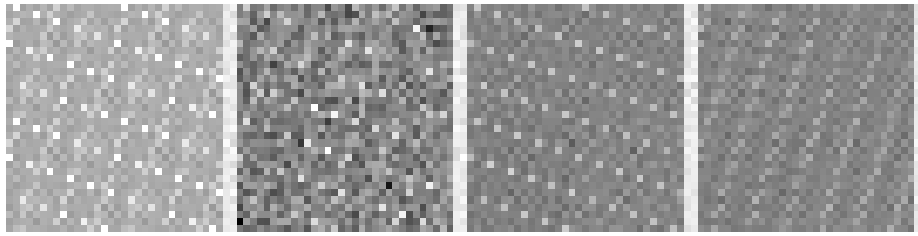

$\mathbf{g}=\mathbf{f}+k_{b} \mathbf{f} \quad \mathbf{g}^{\prime}=\mathbf{g}+$ noise $\quad \mathbf{g}^{\prime} P_{6}$

$\mathbf{g}^{\prime} P_{7}$

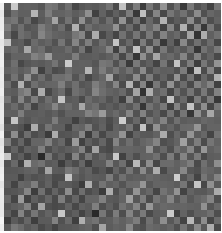

$\mathbf{g}^{\prime} P_{6}+\mathbf{g}^{\prime} P_{7}$

The first image in Figure 40 is generated by adding to $\mathbf{f}$ its translation by $k_{b}$.

The results of applying $P_{6}$ and $P_{7}$ in noise are displayed.

Figure 41. Results of filtering in noise, $N=32$

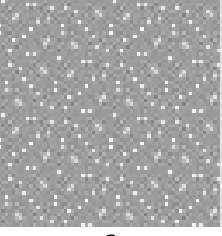

f

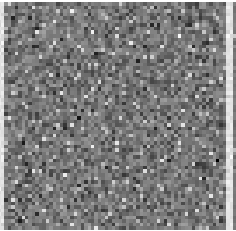

$\mathbf{f}^{\prime}=\mathbf{f}+$ noise

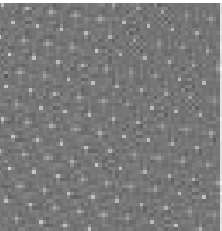

$\mathbf{f}^{\prime} P_{6}$

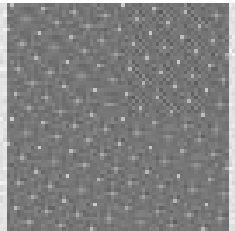

$\mathrm{g} P_{7}$

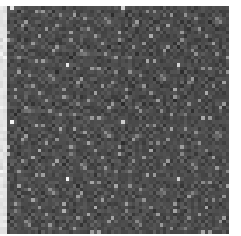

$\mathbf{f}^{\prime} P_{6}+\mathbf{g} P_{7}$ 


\section{References}

All the material presented in this paper has been reproduced from the text Group Filters and Image Processing, M. An and R. Tolimieri, Psypher Press, 2003, with explicit permissions from the authors and the publisher.

1 M. An, R. Tolimieri, B. York, Technical report, AF \#F49620-98C-0043, May, 2002.

2 P. Diaconis, D. Rockmore, "Efficient computation of the Fourier transform on finite groups," J. of AMS 3(2), 297-332, 1990.

3 R. Foote, D. Healy, G. Mirchandani, T. Olson and D. Rockmore, "A wreath product group approach to signal and image processing: Part I Multiresolution analysis," IEEE Trans. in SP, 48(1), 2000, pp. 102-132.

4 R. Holmes, "Signal processing on finite groups," Technical Report 873, MIT Lincoln Laboratory, 1990.

5 M. Karpovski and E. Trachtenberg, "Filtering in a communication channel by Fourier transforms over finite groups," Spectral techniques and fault detection, M. Karpovski, ed., Academic Press, 1985.

6 F.J. MacWilliams, "Codes and ideals in group algebras," Combinatorial mathematics and its applications, 317-328, R.C. Bose and T.A. Dowlin, eds., University of North Carolina Press, 1969.

7 D. Maslen and D. Rockmore, "Generalized FFTs" DIMACS Ser. in Disc. Math. and Theor. Comp. Sci., 28, 183-237, L. Finkelstein and W. Kantor, eds., 1997.

8 R. Tolimieri, M. An, Time-Frequency Representaions, Birkhauser, 1998. 